\title{
The quasilinear theory in the approach of long-range systems to quasi-stationary states
}

\author{
Alessandro Campa ${ }^{1}$ and Pierre-Henri Chavanis ${ }^{2}$ \\ ${ }^{1}$ Complex Systems and Theoretical Physics Unit, \\ Health and Technology Department, Istituto Superiore di Sanità, \\ and INFN Roma1, Gruppo Collegato Sanità, 00161 Roma, Italy \\ 2 Laboratoire de Physique Théorique, Université Paul Sabatier, \\ 118 route de Narbonne 31062 Toulouse, France
}

\begin{abstract}
We develop a quasilinear theory of the Vlasov equation in order to describe the approach of systems with long-range interactions to quasi-stationary states. We derive a diffusion equation governing the evolution of the velocity distribution of the system towards a steady state. This steady state is expected to correspond to the angle-averaged quasi-stationary distribution function reached by the Vlasov equation as a result of a violent relaxation. We compare the prediction of the quasilinear theory to direct numerical simulations of the Hamiltonian Mean Field model, starting from an unstable spatially homogeneous distribution, either Gaussian or semi-elliptical. In the Gaussian case, we find that the quasilinear theory works reasonably well for weakly unstable initial conditions (i.e. close to the critical energy $\epsilon_{\mathrm{c}}=3 / 4=0.75$ ) and that it is able to predict the energy $\epsilon_{\mathrm{t}} \simeq 0.735$ marking the out-of-equilibrium phase transition between unmagnetized and magnetized quasi-stationary states. Similarly, the quasilinear theory works well for energies close to the instability threshold of the semi-elliptical case $\epsilon_{c}^{*}=5 / 8=0.625$, and it predicts the out-of-equilibrium transition at $\epsilon_{t} \simeq 0.619$. In both situations, at energies lower than the out-of-equilibrium transition the quasilinear theory works less well, the disagreement with the numerical simulations increasing by decreasing the energy. In that case, we observe, in agreement with our previous numerical study [A. Campa and P.-H. Chavanis, Eur. Phys. J. B 86, 170 (2013)], that the quasi-stationary states are remarkably well fitted by polytropic distributions (Tsallis distributions) with index $n=2$ (Gaussian case) or $n=1$ (semi-elliptical case). In particular, these polytropic distributions are able to account for the region of negative specific heats in the out-of-equilibrium caloric curve, unlike the Boltzmann and Lynden-Bell distributions.

PACS numbers:
\end{abstract}




\section{INTRODUCTION}

Systems with long-range interactions exhibit peculiar properties both at equilibrium and out-of-equilibrium. Among the equilibrium features that are not present in short-range systems, probably the most striking are the ensemble inequivalence and the negative specific heat in the microcanonical ensemble. Even richer is the phenomenology one observes out-of-equilibrium: long-lived quasi-stationary states (QSSs), non-mixing behavior, phase transitions between non-Boltzmannian distributions, etc [1 4]. Many results have been established, or illustrated, in the context of a toy model called the Hamiltonian Mean Field (HMF) model [5]. Although progress has constantly been made in the last couple of decades, there are still points very poorly understood. One of these issues concerns the approach to QSSs.

In the statistical dynamics study of long-range systems, one of the main tools is the Vlasov equation that represents the interaction through a mean-field. For this reason the Vlasov equation describes a "collisionless" dynamics. It can be shown that this is a very good approximation that improves more and more when the number of components increases (the Vlasov equation becomes essentially exact when the number of particles $N \rightarrow+\infty$ ). More precisely, the larger the system the larger the time range for which the true dynamics can be studied with the Vlasov equation. Eventually, "collisional" effects, i.e. finite $N$ effects, will take over, driving the system towards Boltzmann-Gibbs equilibrium. However, in some cases, especially in the astrophysical context, the time scale of these "collisional" effects can be orders of magnitudes larger that the age of the Universe. It is therefore important to characterize the various stages of the Vlasov dynamics. This equation admits both an infinite number of conserved quantitites (the Casimirs) and an infinite number of stationary states. The QSS reached by the system after an initial transient is just one of these stationary states, obviously one which is stable with respect to perturbations. The infinite number of stationary states of the Vlasov equation makes the prediction of which one is selected by the system very difficult [6, 7]. Actually, it is fair to say that, theoretically, this is still a completely unsolved problem.

The initial state is in general a non-stationary, or unstable stationary, state of the Vlasov equation. In that case, the system evolves very rapidly, with the dynamics governed by the Vlasov equation, towards a QSS. Simulations show that, as one could argue on the 
basis of the existence of an infinite number of stationary states, the selected QSS strongly depends on the initial state of the system. A predictive theory was built by Lynden-Bell [8] in an astrophysical context. According to this theory, the QSS reached by the system is the one that maximizes an entropy functional while preserving the energy and all the Casimirs. Although perfectly defined, this problem is amenable to a computable solution only when the distribution of the initial state has a small number of phase levels, i.e., it is piecewise constant. However, even when the computation is possible, the comparison with the simulations shows that the prediction of the Lynden-Bell theory works only in some cases. The explanation of its failure ${ }^{1}$ has to be sought in the absence of a complete mixing of the dynamics (under given constraints), a property that is implicitly assumed in the theory [7. Even worse, in self-gravitating systems, e.g. elliptical galaxies, the Lynden-Bell distribution has infinite mass, thus it cannot represent the actual QSS of the system.

In the attempt to find alternatives, we have proposed [11] to compare the QSS found in numerical simulations of the HMF model with polytropic distributions (sometimes called Tsallis distributions [12]). These distributions are critical points of functionals of the oneparticle distribution function that generalize the usual Boltzmann entropy. This by itself does not give to polytropes a privileged status with respect to other possible stationary states of the Vlasov equation. The reasons to employ them can be summarized as follows. They have been introduced long ago in astrophysics, where they are called stellar polytropes, in the attempt to describe the non-Boltzmannian distributions observed [13]. Being the critical points (at fixed particle number and energy) of functionals of the form $S[f]=$ $-\int \mathrm{d} \mathbf{x} \mathrm{d} \mathbf{p} C(f)$, where $C(f)$ is a convex function, they determine distributions of the form $f(e)$, where $e$ is the individual energy, with $f^{\prime}(e)<0$ [14]. The stability of these Vlasov stationary states has to be determined in each case (see, e.g., the comments in this respect in Ref. [15, 16]). The function $C(f)$ corresponding to the Boltzmann entropy is $C(f)=$ $-f \ln f$. Under suitable assumptions the function $C(f)=f^{q} /(1-q)$ that gives rise to polytropic distributions can be obtained from a modification of the Lynden-Bell theory that

\footnotetext{
${ }^{1}$ Although we shall focus in this paper on situations where the Lynden-Bell theory does not provide a good description of the QSSs, we would like to emphasize that there exist situations where the Lynden-Bell theory works remarkably well and is able to predict the existence of out-of-equilibrium phase transitions and re-entrant phases that would not have been detected without the help of this theory; see in particular Refs. [9, 10] and Fig. 36 of Ref. [1] for successful predictions of the Lynden-Bell theory.
} 
takes into account the absence of complete mixing [16, 17]. In a numerical study [11], we have shown that in some cases in which the prediction of the Lynden-Bell theory fails to obtain the distribution of the QSSs reached by the system after the violent relaxation, polytropic distributions are a good approximation of the QSSs. However, we have found that the quality of the approximation worsens when the initial distribution is only weakly unstable.

In the case of weak instability of initially homogeneous distributions, another type of approximation could be more suitable, i.e. the quasilinear (QL) approximation. ${ }^{2}$ This approach is based on the assumption that, although the initial distribution is not Vlasov stable, nevertheless its evolution towards a Vlasov stable stationary state is such that it is all the time only slightly inhomogeneous. The assumption will be made more precise below. In this paper we have tested the validity of the QL theory in the HMF model, motivated by the fact that the simulations performed in [11] to study the polytropic approximation have shown that, when the initial distribution is only weakly unstable and the polytropic approximation is not good, the QSS is only weakly inhomogeneous. Therefore, we make the hypothesis that the distribution is only slightly inhomogeneous all the time and compare the prediction of the QL theory to the results of numerical simulations.

In Section II we give the derivation of the QL approximation for a generic mean field system composed of particles moving on a circle. In Section III, we apply the theory to the HMF model, where the particular form of the interaction allows a simplification of the expressions. In Sections IV, V and VI we show the results for a Gaussian and semi-elliptical initial condition and determine the domain of validity of the QL theory and the polytropic fit.

\section{THE QL APPROXIMATION}

We describe here the derivation of the QL approximation for the evolution of the oneparticle distribution function as determined from the Vlasov equation. In view of the application to the HMF model, we consider a generic one dimensional system on the circle, i.e.,

\footnotetext{
${ }^{2}$ The QL theory is well-known in plasma physics for the Coulombian interaction [18. We apply it here to a new situation, namely the HMF model, in which the interaction between particles is attractive.
} 
a system with Hamiltonian

$$
H=\sum_{i=1}^{N} \frac{p_{i}}{2}+\frac{1}{N} \sum_{1 \leq i<j \leq N} \sum_{j} V\left(\theta_{i}-\theta_{j}\right),
$$

where $\theta \in[0,2 \pi]$, and the potential $V(\theta)$, which has $2 \pi$ periodicity, is supposed to be continuous (the mass of the particles has been put equal to 1 without loss of generality). The Vlasov equation for the one-particle distribution function $f(\theta, p, t)$ is

$$
\frac{\partial f(\theta, p, t)}{\partial t}+p \frac{\partial f(\theta, p, t)}{\partial \theta}-\frac{\partial \Phi(\theta, t ; f)}{\partial \theta} \frac{\partial f(\theta, p, t)}{\partial p}=0
$$

where the mean field potential is given by

$$
\Phi(\theta, t ; f)=\int \mathrm{d} p^{\prime} \int_{0}^{2 \pi} \mathrm{d} \theta^{\prime} V\left(\theta-\theta^{\prime}\right) f\left(\theta^{\prime}, p^{\prime}, t\right) .
$$

For large long-range systems with $N \gg 1$ the Vlasov equation is a very good approximation for times of order $N$ [1-4]. Functions $f_{0}(p)$ that depend only on $p$ are particular stationary solution of the Vlasov equation. ${ }^{3}$ The stability of these stationary solutions is studied by putting $f(\theta, p, t)=f_{0}(p)+f_{1}(\theta, p, t)$ and linearizing the Vlasov equation around $f_{0}(p)$, i.e., keeping only the terms at most linear in $f_{1}$. We then obtain the following linear equation for $f_{1}(\theta, p, t)$ :

$$
\frac{\partial f_{1}(\theta, p, t)}{\partial t}+p \frac{\partial f_{1}(\theta, p, t)}{\partial \theta}-\frac{\partial \Phi\left(\theta, t ; f_{1}\right)}{\partial \theta} \frac{\partial f_{0}(p)}{\partial p}=0
$$

which is valid as long as $f_{1}(\theta, p, t) \ll f_{0}(p)$. This is the linearized Vlasov equation. The proper frequencies $\omega$ of the dynamics determined by this equation can be studied by inserting solutions where the time dependence is of the form $\mathrm{e}^{-i \omega t}$. The function $f_{0}(p)$ is stable if all the solution for $\omega$ have a non positive imaginary part. In that case, the distribution function $f_{0}(p)$ can change only because of the effects of "collisions" (finite $N$ effects) that are not considered in the Vlasov equation [4, 19]. We recall that in the case of one dimensional systems the time range of validity of the Vlasov equation grows like $N$ (for inhomogeneous systems) or with a higher power of $N$ (for homogeneous systems) [4, 19]. If, on the contrary, some of the frequencies have a positive imaginary part, $f_{0}(p)$ is Vlasov unstable, and the distribution $f(\theta, p, t)$ will depart from $f_{0}(p)$ rapidly.

\footnotetext{
${ }^{3}$ It is easy to see that the mean field potential $\Phi$ is constant in $\theta$ for a uniform distribution, i.e., a function $f$ that does not depend on $\theta$.
} 
There is an approximation that can work if the positive imaginary parts of the proper frequencies are small enough (the scale with which to measure this smallness will be precised later): the so-called QL approximation. We first derive the corresponding equations, then we describe their solutions, and finally we explain in which case one can expect that the approximation is good. We begin by defining the angle average of the distribution $f(\theta, p, t)$ :

$$
f_{0}(p, t)=\frac{1}{2 \pi} \int_{0}^{2 \pi} \mathrm{d} \theta f(\theta, p, t) .
$$

The angle averaged distribution is then used to define $f_{1}(\theta, p, t)$ by

$$
f(\theta, p, t)=f_{0}(p, t)+f_{1}(\theta, p, t) .
$$

Clearly the angle average of $f_{1}$ is zero. Substituting Eq. (6) in the Vlasov equation (2) we have

$$
\frac{\partial f_{0}(p, t)}{\partial t}+\frac{\partial f_{1}(\theta, p, t)}{\partial t}+p \frac{\partial f_{1}(\theta, p, t)}{\partial \theta}-\frac{\partial \Phi\left(\theta, t ; f_{1}\right)}{\partial \theta}\left(\frac{\partial f_{0}(p, t)}{\partial p}+\frac{\partial f_{1}(\theta, p, t)}{\partial p}\right)=0
$$

We can derive an equation for the time variation of $f_{0}$ by taking the angle average of the last equation. We obtain

$$
\frac{\partial f_{0}(p, t)}{\partial t}-\frac{1}{2 \pi} \frac{\partial}{\partial p} \int_{0}^{2 \pi} \mathrm{d} \theta \frac{\partial \Phi\left(\theta, t ; f_{1}\right)}{\partial \theta} f_{1}(\theta, p, t)=0
$$

Using this equation to substitute the time derivative of $f_{0}$ in Eq. (7), we have

$$
\begin{aligned}
\frac{\partial f_{1}(\theta, p, t)}{\partial t} & +p \frac{\partial f_{1}(\theta, p, t)}{\partial \theta}-\frac{\partial \Phi\left(\theta, t ; f_{1}\right)}{\partial \theta}\left(\frac{\partial f_{0}(p, t)}{\partial p}+\frac{\partial f_{1}(\theta, p, t)}{\partial p}\right) \\
& +\frac{1}{2 \pi} \frac{\partial}{\partial p} \int_{0}^{2 \pi} \mathrm{d} \theta \frac{\partial \Phi\left(\theta, t ; f_{1}\right)}{\partial \theta} f_{1}(\theta, p, t)=0 .
\end{aligned}
$$

Let us now suppose that the following disequality is satisfied during the time evolution

$$
f_{1}(\theta, p, t) \ll f_{0}(p, t)
$$

Then Eq. (9) can be approximated by keeping only the first order terms in $f_{1}$, i.e.

$$
\frac{\partial f_{1}(\theta, p, t)}{\partial t}+p \frac{\partial f_{1}(\theta, p, t)}{\partial \theta}-\frac{\partial \Phi\left(\theta, t ; f_{1}\right)}{\partial \theta} \frac{\partial f_{0}(p, t)}{\partial p}=0
$$

which is a linear equation in $f_{1}$ similar to Eq. (4), but in which $f_{0}$ is not constant in time. The procedure now is to write the solution of this linear equation in $f_{1}$, which depends on 
$f_{0}$, and to insert it in Eq. (8), to obtain a closed equation for $f_{0} \cdot{ }^{4}$ We remark the conceptual difference between Eq. (4) on the one hand, and the couple of equations given by Eqs. (8) and (11) on the other hand. In the former case $f_{0}(p)$ is a stationary solution of the Vlasov equation (2) and, as such, it is by definition constant in time even if from the proper frequencies of Eq. (4) we could find that it is unstable. In the latter case, even if solving Eq. (11) we find that $f_{1}(\theta, p, t)$ does not have exponential growth, in general $f_{0}(p, t)$ defined by Eq. (8) depends on time.

To analyze the solution of Eq. (11) we first consider Eq. (44). Then, we generalize our results to Eq. (11). The solution of Eq. (4) is best obtained by decomposing $f_{1}(\theta, p, t)$ in Fourier components. To study its $k$-th Fourier component, we substitute in Eq. (4) the plane wave expressions

$$
\begin{aligned}
f_{1}(\theta, p, t) & =a_{k}(p) \mathrm{e}^{i(k \theta-\omega t)}, \\
\Phi(\theta, t) & =b_{k} \mathrm{e}^{i(k \theta-\omega t)} .
\end{aligned}
$$

As usual, the physically meaningful solutions are, separately, the real and the imaginary parts of these expressions. The wavenumber $k$ takes all integer values from $-\infty$ to $+\infty$. Since $f_{1}(\theta, p, t)$ and $\Phi(\theta, t)$ are real, in their Fourier expansion (as in Eq. (30) below) there will be both components with $k$ and $-k$. Substituting Eq. (12) and Eq. (13) in Eq. (4) we have

$$
-i \omega a_{k}(p)+i p k a_{k}(p)-i k b_{k} \frac{\partial f_{0}}{\partial p}=0 .
$$

Obviously $b_{k}$ and $a_{k}(p)$ are related. Using the Fourier decomposition of the potential $V(\theta)$

$$
V(\theta)=\frac{1}{2 \pi} \sum_{k=-\infty}^{\infty} V_{k} \mathrm{e}^{i k \theta}
$$

\footnotetext{
${ }^{4}$ We note the formal similarity between Eqs. (8) and (11) and those arising in the kinetic theory of systems with long-range interactions leading to the Lenard-Balescu equation (see Eqs. (12) and (13) in 19]). Both are based on a "quasilinear approximation". However, there are crucial physical differences. We are considering here the collisionless evolution $(N \rightarrow+\infty)$ of a Vlasov unstable distribution function while the Lenard-Balescu kinetic theory is concerned with the collisional evolution (induced by finite $N$ effects) of a Vlasov stable distribution. Thus, our kinetic theory is based on the Vlasov equation (2) instead of the Klimontovich equation (see Eq. (3) in [19]). Our kinetic theory is also different from the kinetic theory of the Vlasov equation (based on a maximum entropy production principle (MEPP) [20] or on another quasilinear approximation [21]) leading to the equilibrium Lynden-Bell distribution. The kinetic theory in [20, 21] implicitly assumes an efficient mixing while our theory, as we shall see, assumes a weak mixing.
} 
where

$$
V_{k}=\int_{0}^{2 \pi} \mathrm{d} \theta V(\theta) \mathrm{e}^{-i k \theta}
$$

we get

$$
b_{k}=V_{k} \int \mathrm{d} p a_{k}(p) .
$$

With the natural assumption $V(-\theta)=V(\theta)$ we have that $V_{k}$ is real and that $V_{-k}=V_{k}$. Substituting Eq. (17) in Eq. (14) this equation becomes

$$
(p k-\omega) a_{k}(p)-k V_{k} \frac{\partial f_{0}}{\partial p} \int \mathrm{d} p^{\prime} a_{k}\left(p^{\prime}\right)=0 .
$$

We have to look for which values of $\omega$ this equation admits solutions $a_{k}(p)$ which are not identically zero. Since this equation is linear, we can freely impose the normalization condition

$$
\int \mathrm{d} p^{\prime} a_{k}\left(p^{\prime}\right)=1
$$

so that the equation becomes

$$
(p k-\omega) a_{k}(p)-k V_{k} \frac{\partial f_{0}}{\partial p}=0
$$

As a matter of fact this equation is satified (for $k \neq 0$ ) for any real value of $\omega$, that we denote by $\omega_{\mathrm{R}}$, by putting

$$
a_{k}\left(p ; \omega_{\mathrm{R}}\right)=k V_{k} \mathrm{P} \frac{\frac{\partial f_{0}}{\partial p}}{p k-\omega_{\mathrm{R}}}+|k| c\left(k, \omega_{\mathrm{R}}\right) \delta\left(p k-\omega_{\mathrm{R}}\right),
$$

where $\mathrm{P}$ denotes that the principal value is taken when integrating over $p$. The normalization condition for $a_{k}\left(p ; \omega_{\mathrm{R}}\right)$ provided by Eq. 19 implies that

$$
c\left(k, \omega_{\mathrm{R}}\right)=1-k V_{k} \mathrm{P} \int \mathrm{d} p \frac{\frac{\partial f_{0}}{\partial p}}{p k-\omega_{\mathrm{R}}} .
$$

Therefore the real values of $\omega$ are not solutions of a dispersion relation, and thus they are not proper frequencies of the linear equation 20 in the usual sense. These solutions are present also when the Fourier component $V_{k}$ is zero; in that case $a_{k}\left(p ; \omega_{\mathrm{R}}\right)$ is a simple delta function and $c\left(k, \omega_{\mathrm{R}}\right)=1$. Let us note that $a_{-k}\left(p,-\omega_{\mathrm{R}}\right)=a_{k}\left(p, \omega_{\mathrm{R}}\right)$. For $k=0$ the only solution of Eq. 20 is $\omega=0$.

Another type of solutions of Eq. 20 can be obtained for particular complex values of $\omega$. They are given by

$$
a_{k}(p ; \omega)=k V_{k} \frac{\frac{\partial f_{0}}{\partial p}}{p k-\omega}
$$


provided the normalization condition $(19)$ is satified. Integrating both sides with respect to $p$ we see that the complex value of $\omega$ must satisfy

$$
D(k, \omega) \equiv 1-k V_{k} \int \mathrm{d} p \frac{\frac{\partial f_{0}}{\partial p}}{p k-\omega}=0
$$

The function $D(k, \omega)$ is called the response dielectric function. There are isolated complex values of $\omega$ such that $D(k, \omega)=0$ (dispersion relation). These are the proper frequencies of Eq. 20). There are two important relations to note. Since $V_{k}=V_{-k}$ is real, then if $D(k, \omega)=0$ we have that also $D\left(k, \omega^{*}\right)=0$ and $D(-k,-\omega)=0$. Therefore, if for a given wavenumber $k$ there is a complex proper frequency $\omega$, there is also the complex conjugate proper frequency $\omega^{*}$, while for the wavenumber $-k$ there will be the proper frequencies $-\omega$ and $-\omega^{*}$. Correspondingly, we have $a_{k}\left(p, \omega^{*}\right)=a_{k}^{*}(p, \omega)$ and $a_{-k}(p,-\omega)=a_{k}(p, \omega)$.

From the Plemelj formula

$$
\lim _{\eta \rightarrow 0^{+}} \frac{1}{x-x_{0} \mp i \eta}=\mathrm{P} \frac{1}{x-x_{0}} \pm i \pi \delta\left(x-x_{0}\right)
$$

we can obtain the value of $D(k, \omega)$ for real values of $\omega$. If $\omega_{\mathrm{R}}$ and $\omega_{\mathrm{I}}$ are the real and imaginary parts of $\omega$, we have

$$
\lim _{\omega_{\mathrm{I}} \rightarrow 0^{ \pm}} D(k, \omega)=1-k V_{k} \mathrm{P} \int \mathrm{d} p \frac{\frac{\partial f_{0}}{\partial p}}{p k-\omega_{\mathrm{R}}} \pm\left.\frac{i \pi}{k} \frac{\partial f_{0}}{\partial p}\right|_{p=\omega_{\mathrm{R}} / k}=c\left(k, \omega_{\mathrm{R}}\right) \pm\left.\frac{i \pi}{k} \frac{\partial f_{0}}{\partial p}\right|_{p=\omega_{\mathrm{R}} / k}
$$

The different limit from above and below the real line shows that Eq. (24) defines two different analytic functions of $\omega$, one in the upper plane and one in the lower plane. The two limits coincide only for the particular values of $\omega_{\mathrm{R}}$ for which the last term in the right-hand side of Eq. (26) is zero, i.e., for $\omega_{\mathrm{R}} / k$ equal to the values of $p$ where $f_{0}(p)$ is minimum or maximum. If it happens that for these particular values $\omega_{\mathrm{R}}$ we also have $c\left(k, \omega_{\mathrm{R}}\right)=0$, then these real frequencies belong to the proper frequencies, since they satisfy $D(k, \omega)=0$. Therefore among the isolated solutions there can also be real $\omega_{\mathrm{R}}$ such that $c\left(k, \omega_{\mathrm{R}}\right)=0$ and $f_{0}^{\prime}\left(\omega_{\mathrm{R}} / k\right)=0$. In this case the normalized $a_{k}\left(p ; \omega_{\mathrm{R}}\right)$ is given by Eq. 23) with $\omega=\omega_{\mathrm{R}}$ and without the necessity of the principal value.

It is possible to show [22] that, for any given $k \neq 0$, the functions $a_{k}\left(p ; \omega_{\mathrm{R}}\right)$ in Eq. 21) and $a_{k}(p ; \omega)$ in Eq. (23) constitute a complete orthogonal system (with the usual scalar product), when $\omega_{R}$ in the former runs over the real axis and $\omega$ in the latter runs over the 
discrete solutions of the dispersion relation $D(k, \omega)=0 .{ }^{5}$ Therefore, any function $h(p)$ can be expanded as

$$
h(p)=\int \mathrm{d} \omega_{\mathrm{R}} \alpha\left(\omega_{\mathrm{R}} ; k\right) a_{k}\left(p ; \omega_{\mathrm{R}}\right)+\sum_{j} \beta_{j}(k) a_{k}\left(p ; \omega_{j}(k)\right)
$$

with proper coefficients $\alpha\left(\omega_{\mathrm{R}} ; k\right)$ and $\beta_{j}(k)$. As just mentioned, the sum runs on the discrete values (generally both complex and real) satifying $D(k, \omega)=0$. Therefore, if the initial value of $f_{1}(\theta, p, t)$ of Eq. (4) is $h(p) \exp (i k \theta)$, the following evolution is given by

$$
f_{1}(\theta, p, t)=\mathrm{e}^{i k \theta}\left[\int \mathrm{d} \omega_{\mathrm{R}} \alpha\left(\omega_{\mathrm{R}} ; k\right) a_{k}\left(p ; \omega_{\mathrm{R}}\right) \mathrm{e}^{-i \omega_{\mathrm{R}} t}+\sum_{j} \beta_{j}(k) a_{k}\left(p ; \omega_{j}(k)\right) \mathrm{e}^{-i \omega_{j}(k) t}\right] .
$$

The corresponding evolution of $\partial \Phi(\theta, t) / \partial \theta$ is

$$
\frac{\partial \Phi(\theta, t)}{\partial \theta}=i k V_{k} \mathrm{e}^{i k \theta}\left[\int \mathrm{d} \omega_{\mathrm{R}} \alpha\left(\omega_{\mathrm{R}} ; k\right) \mathrm{e}^{-i \omega_{\mathrm{R}} t}+\sum_{j} \beta_{j}(k) \mathrm{e}^{-i \omega_{j}(k) t}\right] .
$$

A general initial value of $f_{1}$ can be expanded in Fourier series as

$$
f_{1}(\theta, p, 0)=\frac{1}{2 \pi} \sum_{k=-\infty}^{\infty} f_{k}(p) \mathrm{e}^{i k \theta}
$$

where the term with $k=0$ can be assumed to be absent (it simply corresponds to a constant in time), and where $f_{-k}=f_{k}^{*}$. Expanding any $f_{k}(p)$ in the form given by Eq. (27), the evolution of $f_{1}$ is given by

$$
f_{1}(\theta, p, t)=\frac{1}{2 \pi} \sum_{k=-\infty}^{\infty} \mathrm{e}^{i k \theta}\left[\int \mathrm{d} \omega_{\mathrm{R}} \alpha\left(\omega_{\mathrm{R}} ; k\right) a_{k}\left(p ; \omega_{\mathrm{R}}\right) \mathrm{e}^{-i \omega_{\mathrm{R}} t}+\sum_{j} \beta_{j}(k) a_{k}\left(p ; \omega_{j}(k)\right) \mathrm{e}^{-i \omega_{j}(k) t}\right] .
$$

The reality of this expression follows from $\alpha\left(-\omega_{\mathrm{R}} ;-k\right)=\alpha^{*}\left(\omega_{\mathrm{R}} ; k\right)$ and $\beta_{j}(-k)=\beta_{j}^{*}(k)$ when the proper frequencies for $k$ and $-k$ are numbered so that for the same $j$ we have $\omega$ for $k$ and $-\omega^{*}$ for $-k$. Finally, the evolution of $\partial \Phi(\theta, t) / \partial \theta$ is

$$
\frac{\partial \Phi(\theta, t)}{\partial \theta}=\frac{1}{2 \pi} \sum_{k=-\infty}^{\infty} i k V_{k} \mathrm{e}^{i k \theta}\left[\int \mathrm{d} \omega_{\mathrm{R}} \alpha\left(\omega_{\mathrm{R}} ; k\right) \mathrm{e}^{-i \omega_{\mathrm{R}} t}+\sum_{j} \beta_{j}(k) \mathrm{e}^{-i \omega_{j}(k) t}\right]
$$

\footnotetext{
${ }^{5}$ Equation (4) is not self-adjoint, and for each solution $a_{k}\left(p ; \omega_{\mathrm{R}}\right)$ or $a_{k}(p ; \omega)$ there is a corresponding solution $\tilde{a}_{k}\left(p ; \omega_{\mathrm{R}}\right)$ or $\tilde{a}_{k}(p ; \omega)$ of the adjoint equation. The orthogonality of the system is expressed by the fact that each given solution $a_{k}$ of Eq. (4) is orthogonal to all the solutions $\tilde{a}_{k}$ of the adjoint equation, except the one corresponding to it [22].
} 
which is also real.

Let us now compute the integral in $\theta$ of the product of $\partial \Phi(\theta, t) / \partial \theta$ and $f_{1}(\theta, p, t)$, the expression that appears in Eq. (8). Multiplying Eqs. (31) and (32) and integrating, we obtain

$$
\begin{aligned}
& \int_{0}^{2 \pi} \mathrm{d} \theta \frac{\partial \Phi(\theta, t)}{\partial \theta} f_{1}(\theta, p, t) \\
= & -i \frac{1}{2 \pi} \sum_{k=-\infty}^{\infty} k V_{k}\left[\int \mathrm{d} \omega_{\mathrm{R}}^{\prime} \alpha\left(\omega_{\mathrm{R}}^{\prime} ;-k\right) \mathrm{e}^{-i \omega_{\mathrm{R}}^{\prime} t}+\sum_{n} \beta_{n}(-k) \mathrm{e}^{-i \omega_{n}(-k) t}\right] \\
\times & {\left[\int \mathrm{d} \omega_{\mathrm{R}} \alpha\left(\omega_{\mathrm{R}} ; k\right) a_{k}\left(p ; \omega_{\mathrm{R}}\right) \mathrm{e}^{-i \omega_{\mathrm{R}} t}+\sum_{j} \beta_{j}(k) a_{k}\left(p ; \omega_{j}(k)\right) \mathrm{e}^{-i \omega_{j}(k) t}\right] . }
\end{aligned}
$$

When $f_{0}(p)$ is stable, there are no complex solutions of Eq. (24). This follows from the fact that its complex solutions come in complex conjugate pairs, and therefore if there are complex solutions there are necessarily unstable modes. ${ }^{6}$ Thus, for a stable $f_{0}(p)$, the term with the sum in Eqs. (31) and 32 is either absent or with only real proper frequencies. Then, $f_{1}(\theta, p, t), \partial \Phi(\theta, t) / \partial \theta$ and the integral of their product, Eq. (33), will not have terms with exponential growth. Actually, the interferences between the real frequencies will give rise to an exponential decay (the Landau damping). ${ }^{7}$ On the other hand, when there are complex proper frequencies, and then with positive imaginary parts (since they come in complex conjugate pairs), there will be terms with exponential growths. In that case, one could approximate the right-hand side of Eq. (33) by keeping only those terms. With the further assumption that for each $k$ there is only one complex proper frequency ${ }^{8}$

\footnotetext{
${ }^{6}$ If $\omega=\omega_{R}+i \omega_{I}$ is a solution of the dispersion relation 24, then $\omega=\omega_{R}-i \omega_{I}$ is also a solution. If the first one is stable the second one is unstable, and vice versa.

${ }^{7}$ We remark that, here, we are treating the linearized Vlasov equation à la Van Kampen [23, i.e., with the Fourier transform in time. This treatment is the most natural one to find the proper frequencies of a linear problem. However, it is also possible to treat the problem à la Landau [24, i.e., with the Laplace transform in time. The latter procedure is more suitable to study an initial value problem and to obtain the collective damped modes that result from the interference effects (in particular the Landau damping). In the treatment with the Laplace transform, the dielectric function is defined by Eq. (24) only in the upper $\omega$ plane; in the rest of the complex plane, it is defined by the analytic continuation. In the treatment à la Landau one sees how the Landau damping stems from the real frequencies of the treatment à la Van Kampen (see, e.g., 18 for mathematical details.)

8 According to the Nyquist theorem [25, 26, for an unstable single humped distribution function $f_{0}(p)$, there is only one unstable mode. In more general cases, if there are several complex proper frequencies, one selects the largest one, i.e., the one that corresponds to the maximum growth rate.
} 
$\omega(k)=\omega_{\mathrm{R}}(k)+i \omega_{\mathrm{I}}(k)$ with $\omega_{\mathrm{I}}(k)>0$ (and its complex conjugate) we obtain

$$
\int_{0}^{2 \pi} \mathrm{d} \theta \frac{\partial \Phi(\theta, t)}{\partial \theta} f_{1}(\theta, p, t) \approx-i \frac{1}{2 \pi} \sum_{k=-\infty}^{\infty} k V_{k}|\beta(k)|^{2} a_{k}(p ; \omega(k)) \mathrm{e}^{-i\left(\omega(k)-\omega^{*}(k)\right) t} .
$$

Using the expression of $a_{k}(p ; \omega)$ from Eq. (23) the previous expression can be transformed in

$$
\int_{0}^{2 \pi} \mathrm{d} \theta \frac{\partial \Phi(\theta, t)}{\partial \theta} f_{1}(\theta, p, t) \approx \frac{1}{2 \pi} \sum_{k>0} k^{2} V_{k}|\beta(k)|^{2} \frac{2 \omega_{\mathrm{I}}(k)}{\left(p k-\omega_{\mathrm{R}}(k)\right)^{2}+\omega_{\mathrm{I}}^{2}(k)} \frac{\partial f_{0}(p)}{\partial p} \mathrm{e}^{2 \omega_{\mathrm{I}}(k) t}
$$

We now consider Eq. (11). For a linear equation with time dependent coefficients there are not proper frequencies in the usual sense, and the solutions are not of the same form as in Eqs. (12) and (13). However, we can try, for a given Fourier component, similar expressions, i.e.

$$
\begin{gathered}
f_{1}(\theta, p, t)=a_{k}(p, t) \mathrm{e}^{i k \theta} \mathrm{e}^{-i \int_{0}^{t} \mathrm{~d} t^{\prime} \omega\left(t^{\prime}\right)}, \\
\Phi(\theta, t)=b_{k}(t) \mathrm{e}^{i k \theta} \mathrm{e}^{-i \int_{0}^{t} \mathrm{~d} t^{\prime} \omega\left(t^{\prime}\right)} .
\end{gathered}
$$

We substitute Eqs. (36) and (37) in Eq. (11) and we neglect the time derivatives of $a_{k}(p, t)$ and $b_{k}(t)$, so that we obtain an equation similar to (14):

$$
-i \omega a_{k}(p, t)+i p k a_{k}(p, t)-i k b_{k}(t) \frac{\partial f_{0}(p, t)}{\partial p}=0
$$

With this adiabatic approximation, in which the time derivatives of $a_{k}(p, t)$ and $b_{k}(t)$ are neglected, the last equation is solved as before, with the only difference that $f_{0}^{\prime}(p, t)$ and thus $\omega(t)$ depend on time, and the time dependence of $a_{k}(p, t)$ is only through $\omega(t)$. Without repeating the whole procedure, we write directly the expression for the integral of the product of $\partial \Phi(\theta, t) / \partial \theta$ and $f_{1}(\theta, p, t)$ in the approximation that for each $k$ there is only one proper frequency with positive imaginary part (see footnote 8 ):

$$
\int_{0}^{2 \pi} \mathrm{d} \theta \frac{\partial \Phi(\theta, t)}{\partial \theta} f_{1}(\theta, p, t) \approx \frac{1}{2 \pi} \sum_{k>0} k^{2} V_{k}|\beta(k)|^{2} \frac{2 \omega_{\mathrm{I}}(k, t)}{\left(p k-\omega_{\mathrm{R}}(k, t)\right)^{2}+\omega_{\mathrm{I}}^{2}(k, t)} \frac{\partial f_{0}(p, t)}{\partial p} \mathrm{e}^{2 \int_{0}^{t} \mathrm{~d} t^{\prime} \omega_{\mathrm{I}}\left(k, t^{\prime}\right)} .
$$

This equation can be written in a different form by defining a diffusion coefficient

$$
D(p, t)=\sum_{k>0} \frac{2 \chi_{k}(t) \omega_{\mathrm{I}}(k, t)}{\left(p k-\omega_{\mathrm{R}}(k, t)\right)^{2}+\omega_{\mathrm{I}}^{2}(k, t)}
$$


with

$$
\frac{\partial \chi_{k}(t)}{\partial t}=2 \omega_{\mathrm{I}}(k, t) \chi_{k}(t)
$$

where some constants have been incorporated in the definition of $\chi_{k}(0)$. Therefore, Eq. (39) becomes

$$
\int_{0}^{2 \pi} \mathrm{d} \theta \frac{\partial \Phi(\theta, t)}{\partial \theta} f_{1}(\theta, p, t) \approx D(p, t) \frac{\partial f_{0}(p, t)}{\partial p} .
$$

Substituting the right-hand side of this expression in Eq. (8) we obtain

$$
\frac{\partial f_{0}(p, t)}{\partial t}=\frac{\partial}{\partial p}\left(D(p, t) \frac{\partial f_{0}(p, t)}{\partial p}\right),
$$

which is of the form of a diffusion equation. This is the QL approximation for the evolution of the angle averaged distribution function $f_{0}(p, t)$.

Let us finally discuss when we expect that this approximation is good, i.e., when Eq. (10) is satisfied throughout the time evolution. If we assume that $f_{0}(p, t)$ is of order 1 , we can

argue that the approximation is good as long as $\chi_{k}^{1 / 2}(t) \ll 1$ for each $k$. This defines the smallness of $\omega_{\mathrm{I}}(k)$ also in relation to the initial values $\chi_{k}(0)$. We note that, in general, we expect that $\omega_{\mathrm{I}}(k)$ decreases in time since the dynamics drives the function $f_{0}(p, t)$ towards a Vlasov (marginally) stable distribution.

\section{APPLICATION TO THE HMF MODEL}

The expressions simplify for the HMF model where the potential is $V(\theta)=1-\cos \theta$. Then, we have

$$
V_{k}=2 \pi \delta_{k, 0}-\pi\left(\delta_{k, 1}+\delta_{k,-1}\right) .
$$

As a consequence, the dielectric function $D(k, \omega)$ has a structure only for $k= \pm 1$, and there is a collective dynamics only for these wavenumbers:

$$
D(k, \omega)=1+\pi k\left(\delta_{k, 1}+\delta_{k,-1}\right) \int \mathrm{d} p \frac{\frac{\partial f_{0}}{\partial p}}{p k-\omega} .
$$

The diffusion coefficient of the QL approximation is then

$$
D(p, t)=\frac{2 \chi(t) \omega_{\mathrm{I}}(t)}{\left(p-\omega_{\mathrm{R}}(t)\right)^{2}+\omega_{\mathrm{I}}^{2}(t)},
$$

where the evolution of $\chi(t)$ is given by Eq. (41). It is sufficient to study the dispersion relation only for $k=1$. It is given by

$$
1+\pi \int \mathrm{d} p \frac{\frac{\partial f_{0}(p, t)}{\partial p}}{p-\omega}=0 .
$$


Things simplify further if we consider a function $f_{0}(p)$ that is initially even in $p$ and with only a single maximum at $p=0$. These properties are conserved by the diffusion equation for $f_{0}(p, t)$ (we will prove this below). It is then easy to show that if there is a proper frequency with a positive imaginary part (unstable case), its real part must be zero. Indeed, we can write the dispersion relation as

$$
1+\pi \int \mathrm{d} p \frac{f_{0}^{\prime}(p, t)\left(p-\omega_{\mathrm{R}}(t)\right)}{\left(p-\omega_{\mathrm{R}}(t)\right)^{2}+\omega_{\mathrm{I}}^{2}(t)}+i \pi \omega_{\mathrm{I}} \int \mathrm{d} p \frac{f_{0}^{\prime}(p, t)}{\left(p-\omega_{\mathrm{R}}(t)\right)^{2}+\omega_{\mathrm{I}}^{2}(t)}=0 .
$$

The real and imaginary parts must be separately equal to zero. However, the last integral is negative (positive) definite if $\omega_{\mathrm{R}}(t)>0(<0)$, and it is zero when $\omega_{\mathrm{R}}(t)=0$. This can be seen as follows: (i) for $\omega_{\mathrm{R}}(t)=0$ it vanishes since $f_{0}^{\prime}(p, t)$ is odd; (ii) for $\omega_{\mathrm{R}}(t)>0$, exploiting that $f_{0}^{\prime}(p, t)$ is odd and that it is negative (positive) for $p>0(<0)$, the sum of the values of the integrand for $p$ and $-p$ is negative; (iii) analogously for $\omega_{\mathrm{R}}(t)<0$ this sum is positive. To be more explicit, consider for example the case $\omega_{\mathrm{R}}(t)>0$. Then, for any $p>0$, we have $\left|p-\omega_{\mathrm{R}}(t)\right|<\left|-p-\omega_{\mathrm{R}}(t)\right|$, i.e., $\left(p-\omega_{\mathrm{R}}(t)\right)^{2}+\omega_{\mathrm{I}}^{2}(t)<\left(-p-\omega_{\mathrm{R}}(t)\right)^{2}+\omega_{\mathrm{I}}^{2}(t)$. Thus, since for $p>0$ one has $f_{0}^{\prime}(p, t)=-f_{0}^{\prime}(-p, t)<0$, the sum of the contributions of $p$ and $-p$ to the last integral in Eq. (48) is negative. Therefore, since we must have $\omega_{\mathrm{R}}(t)=0$, the dispersion relation becomes ${ }^{9}$

$$
1+\pi \int \mathrm{d} p \frac{p f_{0}^{\prime}(p, t)}{p^{2}+\omega_{\mathrm{I}}^{2}(t)}=0
$$

and the diffusion coefficient

$$
D(p, t)=\frac{2 \chi(t) \omega_{\mathrm{I}}(t)}{p^{2}+\omega_{\mathrm{I}}^{2}(t)} .
$$

Summarizing, for the HMF model, the integration of the diffusion equation 43 is performed with $D(p, t)$ given by Eq. (50), where $\omega_{\mathrm{I}}(t)$ is the unique positive solution of Eq. (49), with $f_{0}(p, t)$ single humped and even, and where the evolution equation for $\chi(t)$ is

$$
\frac{\mathrm{d} \chi(t)}{\mathrm{d} t}=2 \omega_{\mathrm{I}}(t) \chi(t)
$$

We remark that Eq. (43) is a diffusion equation with a time (and velocity) dependent diffusion coefficient given, for the HMF model, by Eq. (50), which is different from 0 as long as $\omega_{\mathrm{I}}(t)$ does not vanish. If it happens that $\omega_{\mathrm{I}}$ vanishes at a certain time, then the solution

\footnotetext{
${ }^{9}$ Another proof of this result is given in Sec. 2.8. of [25]. Considering an unstable single humped symmetric distribution $f_{0}(p)$, and using the Nyquist theorem, one can show that the dispersion relation (48) has a unique solution with $\omega_{I}>0$; it is such that $\omega_{R}=0$.
} 
of the diffusion equation reaches a stationary state. Actually, as already noted, we expect that the diffusion equation will drive the function $f_{0}(p, t)$ towards a stationary state by monotonically decreasing $\omega_{\mathrm{I}}(t)$ up to 0 . We do not have a general proof of this, but we can show that, if at time $t=0$ the imaginary pulsation $\omega_{\mathrm{I}}(t)$ is positive, it has to tend to 0 for increasing time [see Eq. (62) below]. We cannot prove that $\omega_{\mathrm{I}}(t)$ decreases monotonically; however, we can give an approximate argument, described in Appendix A, to show that this is likely to be the case. The argument is based on the result presented below in Eq. (59), and on the fact, found in the numerical integration of the diffusion equation and shown in the next Section, that the function $f_{0}(p, t)$ keeps approximately the same functional form while it varies as driven by the diffusion equation.

It is useful to make the following evaluation. If we suppose that, at a given time $t, f_{0}(p, t)$ is a Gaussian

$$
f_{0}(p, t)=\frac{1}{2 \pi} \sqrt{\frac{\beta(t)}{2 \pi}} \mathrm{e}^{-\frac{1}{2} \beta(t) p^{2}}
$$

which is normalized such that $\int_{-\infty}^{+\infty} f_{0}(p, t) d p=1 / 2 \pi$, then the dispersion relation 49 becomes

$$
1-\sqrt{\frac{\beta^{3}}{8 \pi}} \int \mathrm{d} p \frac{p^{2} \mathrm{e}^{-\frac{1}{2} \beta p^{2}}}{p^{2}+\omega_{\mathrm{I}}^{2}}=0,
$$

where we have not indicated the time dependence of $\beta$ and $\omega_{\mathrm{I}}$. The integral in the last expression can be expressed in term of the error function

$$
\operatorname{erf}(x)=\frac{2}{\sqrt{\pi}} \int_{0}^{x} \mathrm{~d} y \mathrm{e}^{-y^{2}}
$$

We have

$$
1-\frac{1}{2} \beta+\omega_{\mathrm{I}} \sqrt{\frac{\pi \beta^{3}}{8}} \mathrm{e}^{\frac{1}{2} \beta \omega_{\mathrm{I}}^{2}}\left[1-\operatorname{erf}\left(\sqrt{\frac{\beta}{2}} \omega_{\mathrm{I}}\right)\right]=0 .
$$

For a general distribution function $f_{0}(p, t)$, we define the kinetic temperature $T(t)$ by

$$
T(t)=\left\langle p^{2}\right\rangle=2 \pi \int_{-\infty}^{+\infty} \mathrm{d} p p^{2} f_{0}(p, t) .
$$

This is the variance of the distribution. For the Gaussian distribution (52), we have $\beta=1 / T$. Therefore, Eq. (55) relates the growth rate $\omega_{I}$ to the temperature $T$ for the Gaussian distribution (52). Let us suppose that at time $t=0$ the function $f_{0}$ is a Gaussian. Its evolution, as determined by Eq. (43), together with Eqs. (50) and (51), will not maintain this structure, i.e., we cannot hope that there will simply be a change with time of the 
parameter $\beta$. However, in our simulations we will see that the true evolution does not depart very much from a Gaussian, at least for the cases considered.

We now prove that an initial $f_{0}(p)$ which is even in $p$ and with only a single maximum at $p=0$ will keep these properties during the dynamics determined by Eq. (43). We have seen that, as long as $f_{0}(p, t)$ is of this form, then $\omega_{\mathrm{R}}(t)=0$, and the diffusion coefficient is given by Eq. (50). This is an even function of $p$, with a single maximum at $p=0$. Therefore, the right-hand side of Eq. (43) is even in $p$. Furthermore, taking the derivative of Eq. (43) with respect to $p$ we have

$$
\frac{\partial}{\partial t} \frac{\partial f_{0}}{\partial p}=\frac{\partial^{2} D}{\partial p^{2}} \frac{\partial f_{0}}{\partial p}+2 \frac{\partial D}{\partial p} \frac{\partial^{2} f_{0}}{\partial p^{2}}+D \frac{\partial^{3} f_{0}}{\partial p^{3}}
$$

If $f_{0}(p, t)$ develops a point $p>0$ with $\frac{\partial f_{0}}{\partial p}=0$, at that moment, by continuity, we will have $\frac{\partial^{2} f_{0}}{\partial p^{2}}=0$ and $\frac{\partial^{3} f_{0}}{\partial p^{3}}<0$. Then, we will have $\frac{\partial}{\partial t} \frac{\partial f_{0}}{\partial p}<0$. This proves that the function $f_{0}(p, t)$ will remain with a single maximum at $p=0$.

We also prove that the average kinetic energy increases with time. In fact, we have

$$
\begin{aligned}
\frac{\partial}{\partial t} \int \mathrm{d} p \frac{p^{2}}{2} f_{0}(p, t) & =\int \mathrm{d} p \frac{p^{2}}{2} \frac{\partial f_{0}(p, t)}{\partial t} \\
=\int \mathrm{d} p \frac{p^{2}}{2} \frac{\partial}{\partial p}\left(D(p, t) \frac{\partial f_{0}(p, t)}{\partial p}\right) & =-\int \mathrm{d} p p D(p, t) \frac{\partial f_{0}(p, t)}{\partial p} .
\end{aligned}
$$

Substituting the expression of the diffusion coefficient (50) in Eq. (58), and using the dispersion relation (49), we obtain

$$
\frac{\partial}{\partial t} \int \mathrm{d} p \frac{p^{2}}{2} f_{0}(p, t)=-2 \chi(t) \omega_{\mathrm{I}}(t) \int \mathrm{d} p \frac{p \frac{\partial f_{0}(p, t)}{\partial p}}{p^{2}+\omega_{\mathrm{I}}^{2}(t)}=\frac{2 \chi(t) \omega_{\mathrm{I}}(t)}{\pi}>0 .
$$

Since

$$
\epsilon_{\text {kin }}(t)=2 \pi \int \mathrm{d} p \frac{p^{2}}{2} f_{0}(p, t)=\frac{1}{2} T(t)
$$

we find that

$$
\frac{d T}{d t}=8 \chi(t) \omega_{\mathrm{I}}(t)>0
$$

and we conclude that the kinetic temperature $T(t)$ increases with time. Therefore, the velocity distribution has the tendency to spread. The increase of the kinetic temperature also qualitatively confirms that the system tends to be less unstable as time goes on.

As anticipated, we can prove that $\omega_{I}(t)$ must necessarily tend to 0 . In fact, the relation

$$
\begin{aligned}
\frac{\partial}{\partial t} \int \mathrm{d} p f_{0}(p, t)^{2} & =2 \int \mathrm{d} p f_{0}(p, t) \frac{\partial f_{0}(p, t)}{\partial t} \\
=2 \int \mathrm{d} p f_{0}(p, t) \frac{\partial}{\partial p}\left(D(p, t) \frac{\partial f_{0}(p, t)}{\partial p}\right) & =-2 \int \mathrm{d} p D(p, t)\left(\frac{\partial f_{0}(p, t)}{\partial p}\right)^{2} \leq 0,
\end{aligned}
$$


is a sort of $H$-theorem for the functional $\Gamma_{2}=\int \mathrm{d} p f_{0}(p, t)^{2}$ similar to the enstrophy in two-dimensional turbulence. ${ }^{10}$ Since $\dot{\Gamma}_{2} \leq 0$ and since $\Gamma_{2}$ is bounded from below by 0 , we conclude that $\dot{\Gamma}_{2} \rightarrow 0$ for $t \rightarrow+\infty$. According to the last equality in Eq. 62, this implies that $D(p, t) \rightarrow 0$ for $t \rightarrow+\infty$ at least for some $p$ 's (note that $f_{0}(p, t)$ cannot become independent on $p$ otherwise it would not be normalizable). Considering the expression (50) of $D(p, t)$ this in turn implies that $\omega_{I}(t)$ must necessarily tend to 0 .

Finally, we derive the approximate expression of the dispersion relation that holds when $\omega_{I}(t)$ is very small. This can be useful in the numerical computations. In fact, as emphasized previously, we expect that during the dynamics $\omega_{\mathrm{I}}(t)$ decreases towards zero, so that $f_{0}(p, t)$ becomes (marginally) Vlasov stable. It is not difficult to see that at first order in $\omega_{\mathrm{I}}(t)$ the dispersion relation (49) (we remind that this expression is valid for an even $f_{0}(p, t)$ with a single maximum at $p=0$ ) becomes for $\omega_{\mathrm{I}}(t) \geq 0:^{11}$

$$
1+\pi \int \mathrm{d} p \frac{f_{0}^{\prime}(p, t)}{p}-\pi^{2} \omega_{\mathrm{I}}(t) f_{0}^{\prime \prime}(0, t)=0
$$

Specializing to the case of the Gaussian (52), Eq. (63) becomes

$$
1-\frac{1}{2} \beta+\sqrt{\frac{\pi \beta^{3}}{8}} \omega_{\mathrm{I}}(t)=0 .
$$

This relation can also be obtained by a power expansion of Eq. (55). From Eq. (63) we obtain

$$
\omega_{\mathrm{I}}(t)=\frac{1}{\pi^{2} f_{0}^{\prime \prime}(0, t)}\left[1+\pi \int \mathrm{d} p \frac{f_{0}^{\prime}(p, t)}{p}\right] .
$$

We remind that the term in square brackets is exactly the expression that one obtains from the Nyquist criterion for the Vlasov stability of a normalized distribution function $f_{0}(p)$ which is even in $p$ and with a single maximum at $p=0$ [25]. Such a function is stable if, and only if, that expression is positive:

$$
f_{0}(p) \quad \text { stable } \quad \Leftrightarrow \quad 1+\pi \int \mathrm{d} p \frac{f_{0}^{\prime}(p, t)}{p}>0
$$

10 This inequality is actually true for any $H$-function [14] of the form $H=-\int C(f) d p$ where $C(f)$ is convex $\left(C^{\prime \prime} \geq 0\right)$ since $\dot{H}=\int \mathrm{d} p D(p, t) C^{\prime \prime}\left(f_{0}(p, t)\right)\left(\frac{\partial f_{0}(p, t)}{\partial p}\right)^{2} \geq 0$.

${ }^{11}$ For $\omega_{\mathrm{I}}(t)<0$, in the last term of the left hand side of Eq. $63 \omega_{\mathrm{I}}(t)$ must be substituted by $\left|\omega_{\mathrm{I}}(t)\right|$. This is consistent with the fact that the expression in Eq. 49 is even in $\omega_{\mathrm{I}}(t)$ (but not differentiable at $\left.\omega_{\mathrm{I}}(t)=0\right)$. Equation (63) is obtained from a straightforward adaptation to the HMF model of the method developed in Sec. 3.7 of [26] for self-gravitating systems. Another, more direct, proof of Eq. (63) is given in Appendix B. 
Then, we expect to have a positive $\omega_{\mathrm{I}}(t)$ if that expression is negative. Since $f_{0}^{\prime \prime}(0, t)<0$ we therefore have a consistent expression.

Remark: We note that the stability criterion (66) can be rewritten as

$$
T_{\text {eff }} \equiv \frac{-1}{2 \pi \int \mathrm{d} p \frac{f_{0}^{\prime}(p, t)}{p}}>\frac{1}{2},
$$

where $T_{\text {eff }}$ is an effective temperature (it coincides with the ordinary temperature when $f_{0}(p)$ is the Gaussian distribution). This formula shows that stability is achieved when the width of the distribution function is sufficiently large, except that the width is not measured by the kinetic temperature (56) but by the effective temperature (67).

\section{RESULTS}

The HMF model exhibits a second order phase transition between a homogeneous unmagnetized phase and a magnetized phase at the critical temperature $T_{c}=1 / 2$, corresponding to the critical energy $\epsilon_{c}=3 / 4=0.75$ (with $\epsilon$ we denote the energy per particle) [4]. The homogeneous phase is thermodynamically stable if, and only if, $T \geq T_{c}$. The critical temperature $T_{c}=1 / 2$ also coincides with the dynamical stability threshold of the homogeneous Gaussian distribution

$$
f_{0}(p)=\frac{1}{2 \pi} \sqrt{\frac{\beta}{2 \pi}} \mathrm{e}^{-\frac{\beta}{2} p^{2}}
$$

Indeed, one finds that in this case the expression in the right hand side of Eq. (66) vanishes for $\beta=\beta_{c}=2$, while it is positive (resp. negative) for $\beta<2$ (resp. $\beta>2$ ). Therefore, a Gaussian distribution at temperature smaller than $1 / 2$, i.e., at energy smaller than 0.75 , is Vlasov unstable.

Other forms of homogeneous distribution functions have other (dynamical) stability thresholds. We will consider a semi-elliptical distribution, given by

$$
f_{0}(p)=\frac{1}{2 \pi^{2} b} \sqrt{2 b-p^{2}} \Theta\left(2 b-p^{2}\right)
$$

where $\Theta(\cdot)$ is the Heaviside step function, and where the parameter $b$ is related to the energy $\epsilon$ by $b=4 \epsilon-2$. A semi-elliptical distribution is a polytrope of index $n=1$ [11, 16]. The stability threshold for this function is $\epsilon_{c}^{*}=\epsilon_{n=1}=5 / 8=0.625$, therefore we will study the dynamics when $\epsilon$ is somewhat smaller than this value. 
We have performed simulations of both the diffusion equation and of the full $N$-body dynamics of the HMF model at several energies smaller than the critical energy for both the cases in which the initial homogeneous distribution is a Gaussian and a semi-ellipse. The analysis and comparison of the results has been as follows. Starting from a Vlasov unstable state, the $N$-body system has a violent relaxation that ends at a QSS, a Vlasov stationary and stable state. Afterwards, the system evolves slowly, driven by finite-size effects, a phase of the dynamics in which we are not interested in this work. The QSS is characterized by a single particle distribution function that in principle will not be homogeneous, depending on both $\theta$ and $p$. On the other hand, the diffusion equation concerns the evolution of the angleaveraged distribution function $f_{0}(p, t)$, and this evolution stops when $f_{0}(p, t)$ reaches a Vlasov stable state for which $\omega_{I}=0$. Therefore, we have compared the angle-averaged distribution function of the $N$-body QSS with the function $f_{0}(p)$ obtained when the evolution of the diffusion equation reaches an end. To characterize this comparison with a single parameter, we have considered the temperature associated to these distributions, i.e., the expected value of $p^{2} / 2$ (since we consider only distributions that are even in $p$, the expected value of $p$ is zero). We note that for the HMF model the kinetic temperature $T$ and the magnetization $m$ of the system, for a given energy, are uniquely related. In fact we have

$$
\epsilon=\frac{1}{2} T+\frac{1}{2}\left(1-m^{2}\right) .
$$

Then, the analysis of the temperature is equivalent to that of the magnetization, i.e., the order parameter. From Eq. (70) we note that the smallest possible energy for a homogeneous initial condition $(m=0)$ is $\epsilon=1 / 2$.

\section{A. Integration of the diffusion equation}

For convenience we rewrite here the diffusion equation

$$
\frac{\partial f_{0}(p, t)}{\partial t}=\frac{\partial}{\partial p}\left(D(p, t) \frac{\partial f_{0}(p, t)}{\partial p}\right),
$$

and the diffusion coefficient for the case of the HMF model with an even and single humped distribution function

$$
D(p, t)=\frac{2 \chi(t) \omega_{\mathbf{I}}(t)}{p^{2}+\omega_{\mathrm{I}}^{2}(t)} .
$$


Eq. (49) determines the value of $\omega_{\mathrm{I}}(t)$, while $\chi(t)$ evolves according to Eq. (51). It follows that the time scale of the dynamics of the diffusion equation depends on the chosen initial value $\chi(0)$. We want to be sure that, independently from this value, provided that it is sufficiently small for the QL approximation to be valid, the final distribution is practically the same. We provide details about this by showing the diffusion equation results for the initial Gaussian distribution.

In Fig. 1 we show the evolution of $\omega_{\mathrm{I}}(t)$ and of $\chi(t)$, according to the diffusion equation, starting from an initial $f_{0}(p, 0)$ given by the Gaussian $(68)$ at temperature $T=1 / \beta=0.38$, corresponding, for $m=0$, to $\epsilon=0.69<\epsilon_{c}$. The left and right panels refer to the integration with initial value $\chi(0)=10^{-6}$ and $\chi(0)=10^{-2}$, respectively.
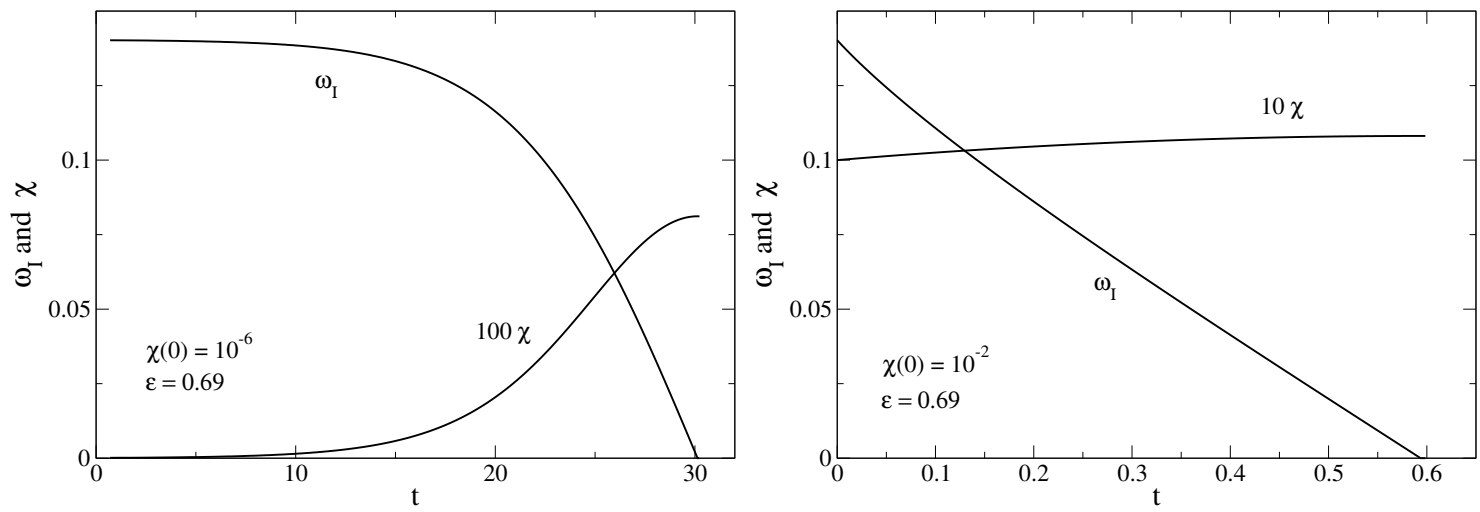

FIG. 1: Evolution of $\omega_{\mathrm{I}}(t)$ and of $\chi(t)$ as determined by the diffusion equation (71) together with Eqs. (72), (49) and (51), for two different initial values $\chi(0)$. The initial distribution function is the Gaussian 68 at $T=1 / \beta=0.38$. Left panel: $\chi(0)=10^{-6}$; right panel: $\chi(0)=10^{-2}$. For graphical convenience in both cases a multiple of $\chi(t)$ is plotted.

We see that the evolutions are qualitatively different. In particular, the convergence towards a stationary solution $\left(\omega_{I}=0\right)$ is more rapid when $\chi(0)$ is large. However, the distribution $f_{0}(p)$ obtained at the end of the evolution does not depend on $\chi(0)$. In the left panel of Fig. 2 we plot this distribution for both cases. It is evident that the two final distributions actually plotted are virtually indistinguishable. ${ }^{12}$ In the right panel we plot the initial and the final distributions. This figure suggests that the form of the final

${ }^{12}$ We note that the final values of $\chi$ are different in the two cases (by a factor 10) even if this difference has been reduced with respect to the initial condition. 

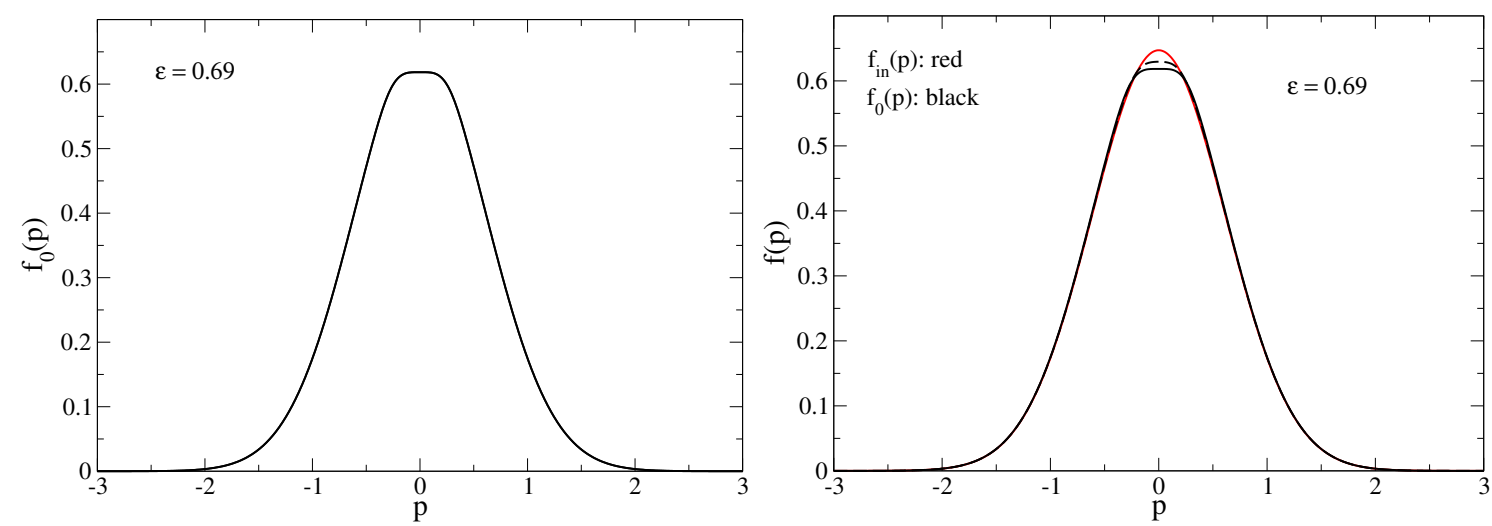

FIG. 2: Left panel: the final distribution functions $f_{0}(p)$, obtained when $\omega_{\mathrm{I}}(t)$ vanishes, for the two cases plotted in Fig. 1. They are indistinguishable. Right panel: the initial and the final distributions. We have also added an intermediate distribution to illustrate the progressive formation of the plateau. The distribution function is normalized such that $\int f(\theta, p) d \theta d p=1$ and $2 \pi \int f(p) d p=1$.

distribution is not very different from a Gaussian, except in the central region, where a sort of flat region seems to have developed. This can be understood as follows. In the core of the distribution $p \ll 1$, the diffusion coefficient can be approximated by $D(p, t) \simeq 2 \chi(t) / \omega_{I}(t)$. Since $\omega_{I}(t)$ decreases to 0 , the diffusion coefficient in the core becomes very large and this implies $\partial f_{0}(p, t) / \partial p \simeq 0$, so that the distribution function in the core forms a plateau. On the other hand, in the tail of the distribution $p \gg 1$, the diffusion coefficient can be approximated by $D(p, t) \simeq 2 \chi(t) \omega_{I}(t) / p^{2}$. Since $\omega_{I}(t)$ decreases to 0 , and since $p \gg 1$, the diffusion coefficient in the tail is very small and this implies $\partial f_{0}(p, t) / \partial t \simeq 0$, so that the distribution function in the tail does not evolve substantially. As a matter of fact, under the assumption that the final distribution has a Gaussian structure apart from a central region, which is flat, it is possible to obtain exactly its parameters. Such an assumption is equivalent to assuming that the diffusion equation gradually develops a flat portion in the central part of the distribution, while maintaining the Gaussian form outside that portion. The possibility to derive exactly this distribution stems from the fact that there are as many equations to satisfy as parameters characterizing the distribution. In fact, the assumed form 
can be written as

$$
\begin{aligned}
f_{\mathrm{mG}}(p) & =C & & |p| \leq p_{1}, \\
& =A \mathrm{e}^{-\beta \frac{p^{2}}{2}} & & |p| \geq p_{1},
\end{aligned}
$$

where the subscript denotes a "modified Gaussian", and where $p_{1} \geq 0$. Continuity implies that $C=A \mathrm{e}^{-\beta \frac{p_{1}^{2}}{2}}$. Therefore there are three parameters to be determined, i.e., $A, \beta$ and $p_{1}$. On the other hand, there are also three equations to satisfy. They are the normalization, the marginal stability of the distribution and the relation expressing the average kinetic energy:

$$
\begin{aligned}
2 \pi \int \mathrm{d} p f_{\mathrm{mG}}(p) & =1, \\
1+\pi \int \mathrm{d} p \frac{f_{\mathrm{mG}}^{\prime}(p)}{p} & =0, \\
2 \pi \int \mathrm{d} p \frac{p^{2}}{2} f_{\mathrm{mG}}(p) & =\epsilon_{\mathrm{kin}},
\end{aligned}
$$

where $\epsilon_{\text {kin }}$ is the final average kinetic energy, determined numerically from the distribution at the end of the integration. In Appendix $\mathrm{C}$ we show how to solve this system of equations for $A, \beta$ and $p_{1}$.
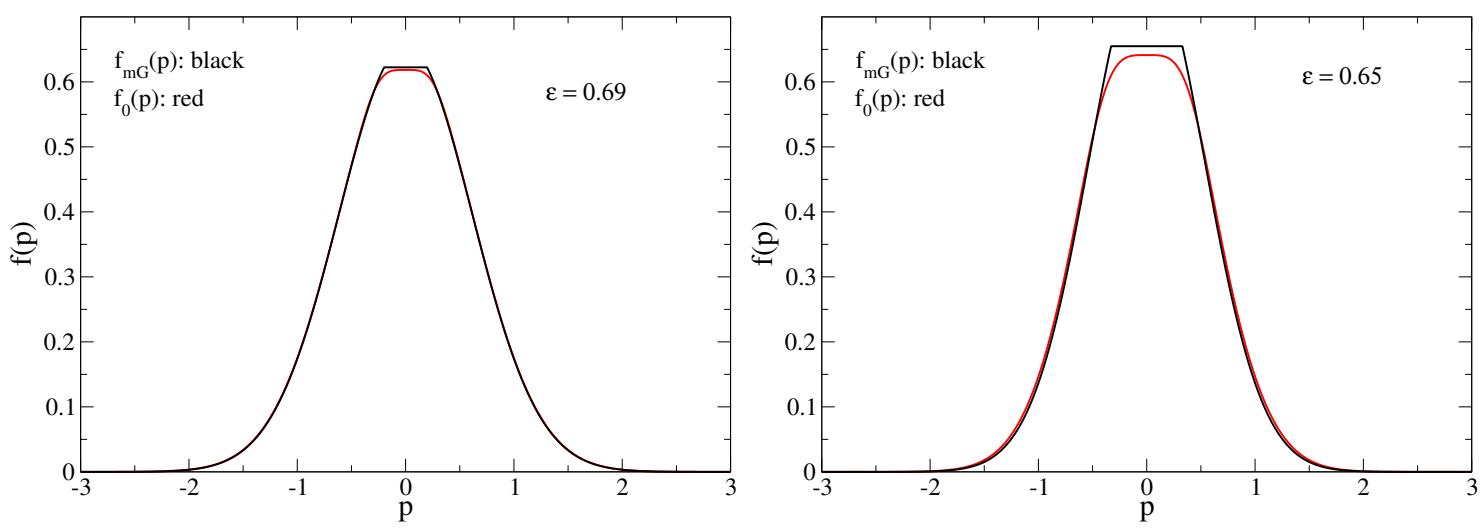

FIG. 3: The final distribution function $f_{0}(p)$ and the modified Gaussian $f_{\mathrm{mG}}(p)$ for energies $\epsilon=0.69$ (left panel) and $\epsilon=0.65$ (right panel). The function $f_{\mathrm{mG}}(p)$ is computed for the same kinetic energy $\epsilon_{\text {kin }}$ resulting from $f_{0}(p)$.

In Fig. 3 we show the comparison, for the energies $\epsilon=0.69$ and $\epsilon=0.65$, between the final distribution of the diffusion equation and the approximation with a modified Gaussian. It is evident that for the larger energy the approximation with a modified Gaussian is rather good, while for the smaller energy there is a clear difference in the region of the plateau. 


\section{B. The $N$-body simulations and the comparison with the results of the diffusion}

equation

In this section, we compare the results of the QL theory with the results of $N$-body simulations. We begin the analysis by showing the evolution of the magnetization for both the diffusion equation and the $N$-body simulations. These simulations have been performed with $N=2^{18}$ particles. As remarked above, for the diffusion equation the magnetization can be computed using Eq. (70), obtaining the kinetic temperature at time $t$ from the variance of $f_{0}(p, t)$ using Eq. (56).

Let us first comment on the comparison between the time scales in the two dynamical evolutions. As explained above and as evidenced in Fig. 1, the time scale of the dynamics of the diffusion equation depends strongly on the initial value $\chi(0)$. This is of course reflected in the temporal evolution of the magnetization.
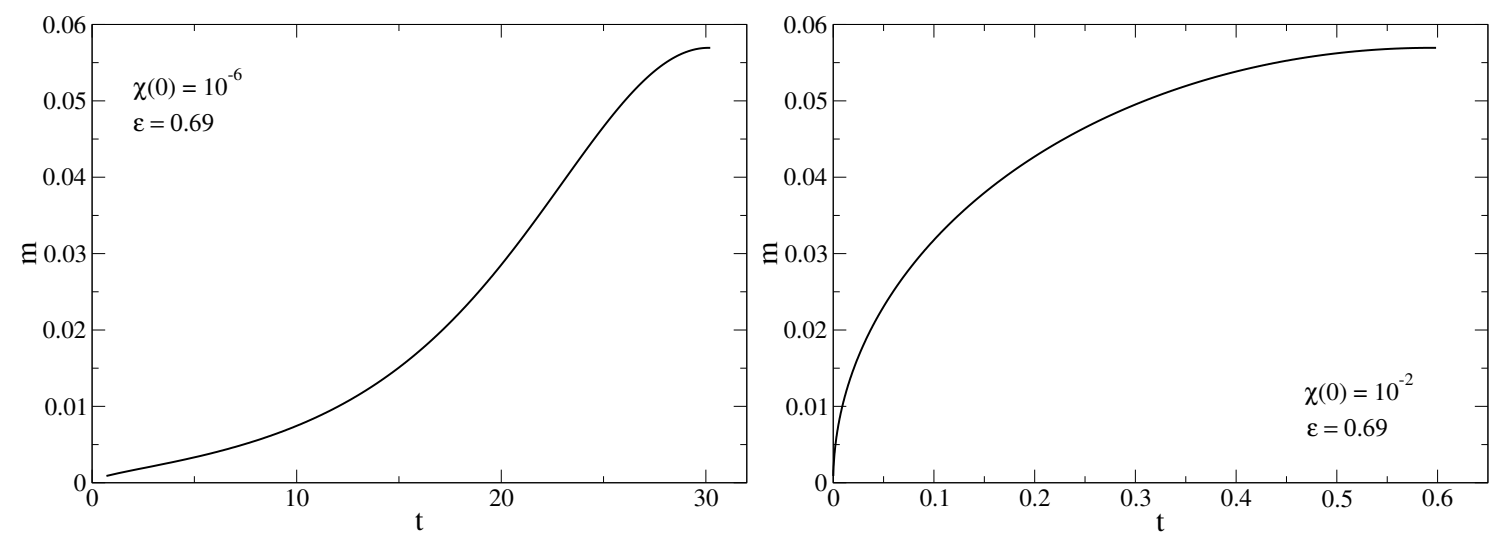

FIG. 4: Magnetization as a function of time from the diffusion equation at energy $\epsilon=0.69$, obtained from the distribution function $f_{0}(p, t)$ using Eqs. 56 and (70). Left panel: $\chi(0)=10^{-6}$. Right panel: $\chi(0)=10^{-2}$.

In Fig. 4, we plot the dynamics of the magnetization as obtained from the diffusion equation for the Gaussian initial condition and energy $\epsilon=0.69$ for the two cases of Fig. 1, i.e., $\chi(0)=10^{-6}$ and $\chi(0)=10^{-2}$. We see that the final magnetization is the same in both cases, as expected from the fact that the final distributions are the same, although the time scale is different. In the diffusion equation, $\chi(0)$ is related to the initial perturbation to the stationary but Vlasov unstable distribution $f_{0}(p, 0)$. It therefore depends on the order of 
magnitude of $f_{1}(\theta, p, 0)$ in Eq. (6). In the $N$-body simulation, this role is played by the finite size effects in the initial conditions determined according to $f_{0}(p, 0)$. From the definition of $\chi(t)$, given implicitly by the comparison of Eqs. (39), 40) and (41), it is clear that the order of magnitude of $\chi(0)$ is that of $f_{1}^{2}(\theta, p, 0)$. In turn, in the $N$-body simulations we expect that the order of magnitude of $f_{1}(\theta, p, 0)$, due to finite size effects, is $N^{-\frac{1}{2}}$. Therefore, we expect that the simulations performed with $N=2^{18}=0.262144 \times 10^{6}$ particles should evolve, as far as the relaxation to the stationary state is concerned, on a time scale similar to that of the diffusion equation with a value of $\chi(0)$ of the order $10^{-6}$. In Fig. 5 we show that this is indeed the case. We stress, however, that the $N$ dependence of this time scale which is due to the dynamical instability of the initial state is expected to behave like $\log N$, contrary to the power law dependence occurring for the slow evolution in the QSS due to collisional effects [4, 27].
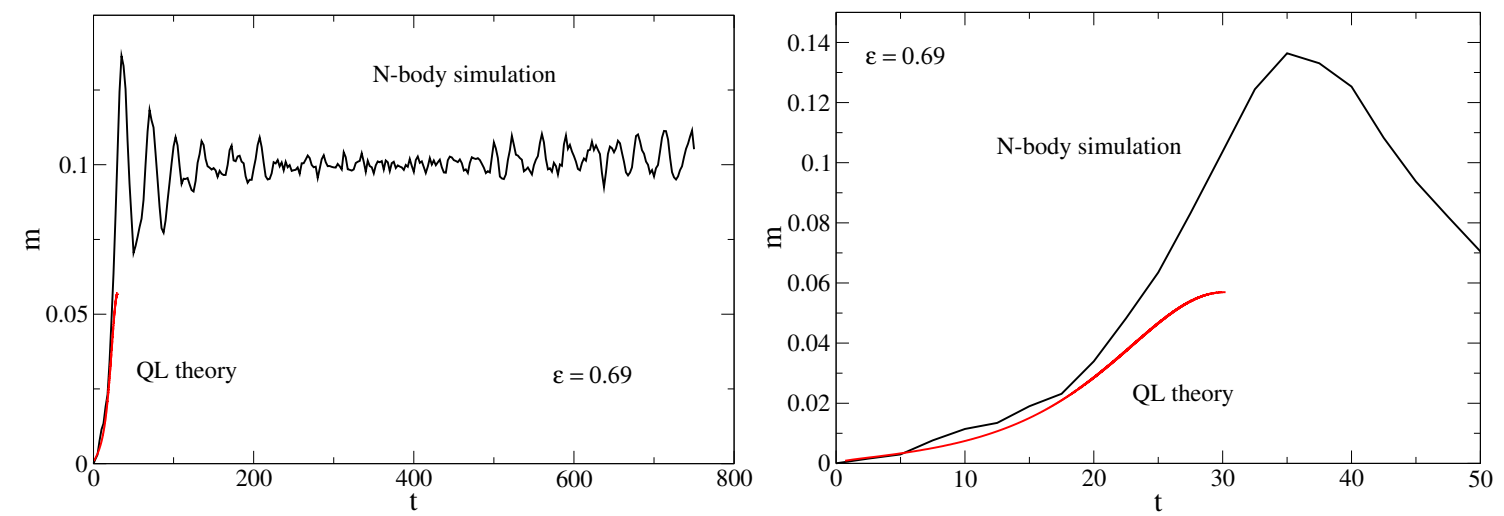

FIG. 5: Magnetization as a function of time at energy $\epsilon=0.69$ for a homogeneous Gaussian velocity initial distribution. Both the $N$-body simulation with $N=2^{18}$ particles (black) and the diffusion equation (red) curves are shown (we have taken $\chi(0)=10^{-6}$ ). Left panel: plot of the magnetization during the whole duration of the simulation. Right panel: plot of the magnetization during the early times of the simulation. In the diffusion equation, the magnetization remains constant at times larger than those plotted since the evolution stops when $\omega_{\mathrm{I}}(t)$ reaches the value 0.

The analysis of Fig. 5 puts in evidence several important things. Firstly, there is no perfect agreement on the final value of the magnetization, which is about 0.057 for the diffusion equation and about 0.10 for the $N$-body simulation. However, these magnetization 
values are comparable, and they are substantially different from that of the BG equilibrium state corresponding to $\epsilon=0.69$ which is about 0.31 . On the other hand, the left panel shows that in the state reached by the $N$-body system at the end of the fast relaxation the magnetization has relevant oscillations. Therefore the system is not in a proper QSS, although one could extend the definition of such a state also to cases that present these oscillations. The QL theory does not account for these oscillations because they appear in the regime where the system is dynamically stable. They may correspond to small perturbations about a stable steady state of the Vlasov equation. A possibility is that these oscillations will decay by Landau damping. Another possibility considered in [11] is that these oscillations will survive during the whole collisionless regime like in the simulations performed by Morita and Kaneko [28]. Apart from the presence of the oscillations, we conclude that, at the level of the final magnetization value, there is a reasonable but not a perfect agreement between the full $N$-body simulation and the approximation represented by the QL theory since, in relative terms, the difference is large. However, we have to consider that for such small values of the magnetization, a very small change in $T$ causes a strong variation in $m$, since the derivative of $m$ with respect to $T$, that can be computed from Eq. (70), diverges for $T \rightarrow 1-2 \epsilon$. As a matter of fact, while the temperature of the QSS of the $N$-body simulation is about 0.390 , the temperature of the final state of the diffusion equation is about 0.383 . At this energy, $T$ is about 0.475 at BG equilibrium. Therefore the relative error in the temperature value of the QSS is much smaller than the error in the magnetization.

Let us now compare the velocity distribution functions of the QSS reached in the $N$-body simulation and the final distribution function of the diffusion equation. In Fig. 6 we show this comparison for the two cases of $\epsilon=0.69$ and $\epsilon=0.725$ for the initial Gaussian distribution. We observe a very good agreement for the energy $\epsilon=0.725$ which is closer to the instability threshold $\epsilon_{c}=3 / 4$. For the case $\epsilon=0.69$, there is a very good agreement in the tail of the distribution but a disagreement concerns the central region of the distribution. The distribution function obtained from the diffusion equation clearly exhibits a central plateau while there is only the hint of a plateau in the distribution function obtained from the $N$-body simulation. We also note that the QL theory predicts that the central distribution function $f(0, t)$ decreases ${ }^{13}$ with respect to its initial value (see Fig. 2), in qualitative agreement with

13 This is consistent with the notion of "coarse-graining". The maximum value of the coarse-grained distri- 

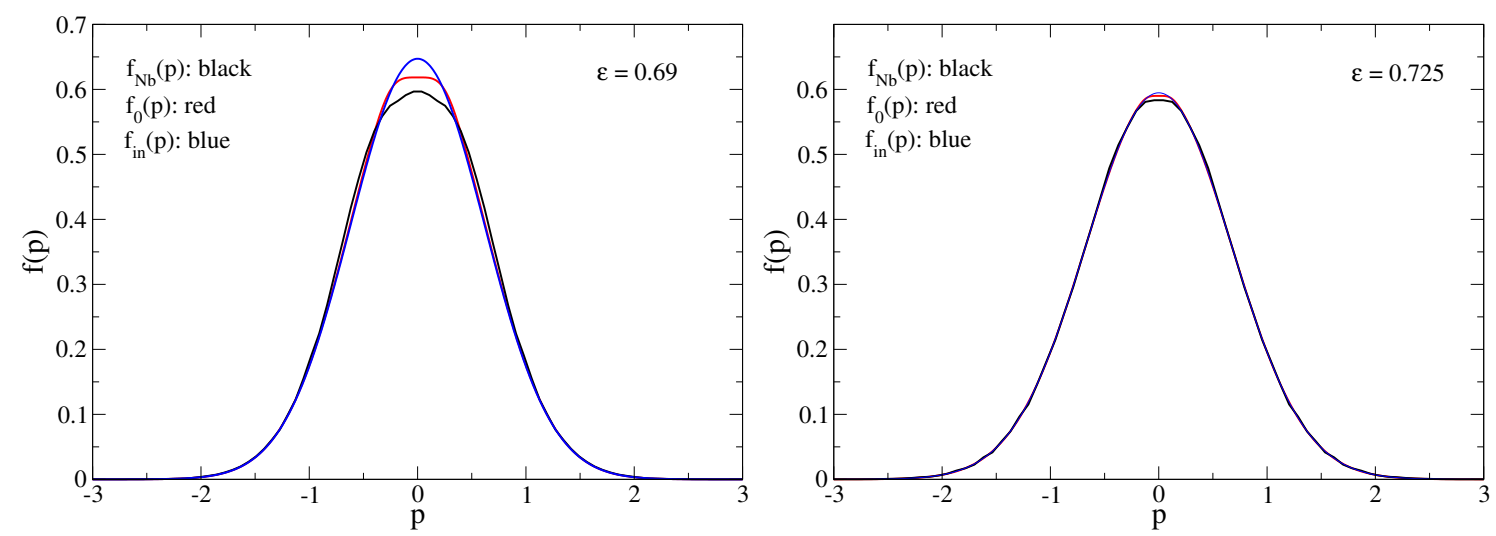

FIG. 6: The final distribution function $f_{0}(p)$ of the diffusion equation and the velocity distribution $f_{\mathrm{Nb}}(p)$ of the QSS of the $N$-body simulation, starting from a Gaussian initial distribution. Left panel: $\epsilon=0.69$. Right panel: and $\epsilon=0.725$. We have also plotted the initial condition $f_{\text {in }}(p)$ for comparison. We emphasize that the initial distribution is Vlasov unstable while the final distribution is (marginally) Vlasov stable even when the profile does not seem to have changed a lot.

the $N$-body simulation, although the decrease is stronger in the $N$-body simulation.

We note that the distribution of the QSS obtained from $N$-body simulations and the distribution predicted by the QL theory have a form very similar to the initial distribution. Actually, the distribution function almost does not change in the tail of the distribution. Nevertheless, the slight change in the central region (core) is crucial because the initial distribution is Vlasov unstable while the QSS and the distribution predicted by the QL theory are Vlasov stable. Therefore, close to the instability threshold, stability can be regained by a very slight modification of the distribution function that affects essentially the core of the distribution (small velocities) while preserving the tail (large velocities).

\section{The nonequilibrium phase transition predicted by the QL theory}

In Ref. [11] we studied the evolution of several unstable homogeneous initial conditions in situations where the Lynden-Bell theory fails to predict with good approximation the QSS reached by the system. We found that, in most of these cases, the QSS could be well

bution can only decreases by phase mixing 8 . 
approximated by a polytropic distribution. Furthermore, the index of the polytrope, even if it was not possible to predict if from first principles, happened to be the same for a given class of initial conditions (e.g. Gaussian, semi-elliptical...) for a wide range of energies. However, we remarked that the polytropic approximation was not good for energies close to the instability threshold pertaining to the class of the initial distribution. We have seen here that, in this case, the QL theory provides a better approximation. In fact, we have seen that the final distribution computed by the QL theory is close to the angle-averaged distribution of the QSS obtained in the $N$-body simulations. Furthermore, the values of the kinetic temperature and magnetization predicted by the QL theory are relatively close to those obtained from the direct $N$-body simulations, even if the quality of the agreement deteriorates if we are too far away from the instability threshold.
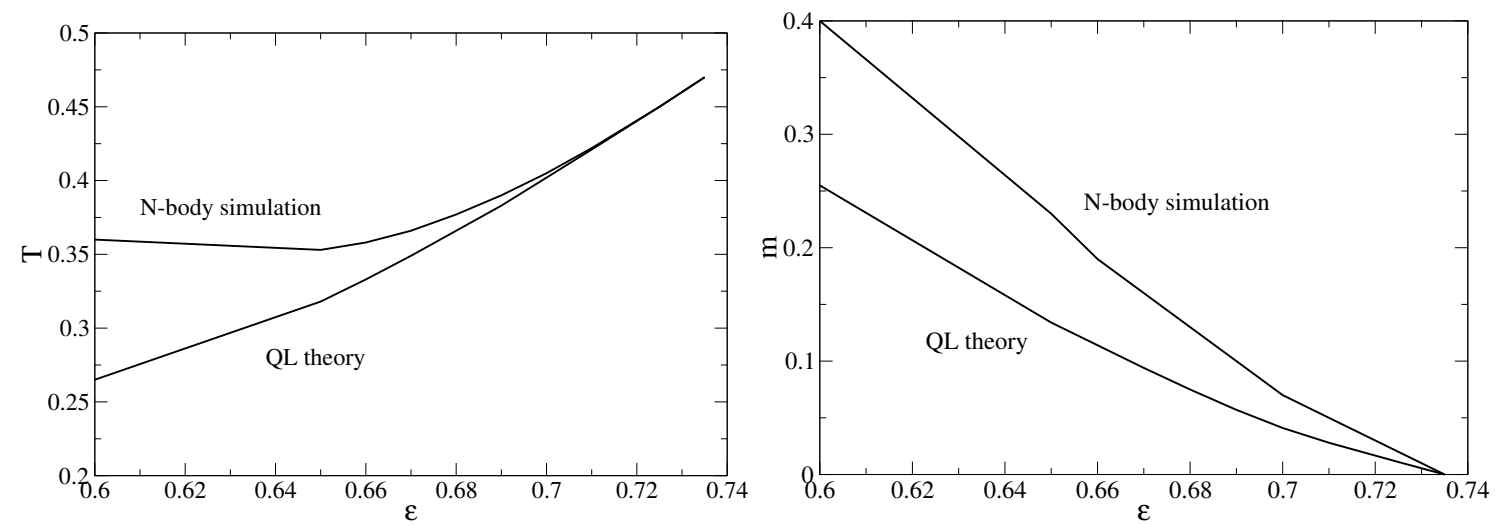

FIG. 7: The kinetic temperature and the magnetization of the stationary state reached by the diffusion equation of the QL theory and that of the QSS reached by the $N$-body simulation, as a function of the energy. The homogeneous initial distribution is a Gaussian in the velocity.

To give an easily grasped quantitative meaning to this agreement/disagreement, we plot in Fig. 7 the kinetic temperature and the magnetization of the final distribution of the QL theory as a function of the energy for the Gaussian initial distribution. The plot also shows the kinetic temperature and the magnetization of the QSS reached in the $N$-body simulation at the same energy. Although the magnetization of the QL theory is different from that of the simulation, the plot shows that the energy of the phase transition from the unmagnetized state $(m=0)$ to the magnetized state $(m \neq 0)$ is well predicted by the $\mathrm{QL}$ theory. We stress that this is to be interpreted as a nonequilibrium phase transition since 
it refers to the magnetization of the out-of-equilibrium QSSs. We can conclude that the QL theory is able to localize this phase transition correctly. A more detailed discussion is provided in the following section.

\section{COMPARISON OF THE QSS WITH THE PREDICTION OF THE QL THE- ORY AND WITH THE POLYTROPIC FIT}

\section{A. Caloric and magnetization curves}

In this section, we compare the caloric curve obtained from direct $N$-body simulations of the HMF model, starting from a homogeneous Gaussian distribution, with the prediction of the QL theory and with the polytropic fit considered in our previous papers [11, 16].

The caloric curve $T(\epsilon)$ is represented in Fig. 8 and the magnetization curve $m(\epsilon)$ is represented in Fig. 9. We recall that, for a given energy $\epsilon$, the kinetic temperature $T$ is related to the magnetization by Eq. (70). Therefore, these two curves are equivalent. The non-magnetized branch $m=0$ in Fig. 9 corresponds to the line $T=2 \epsilon-1$ in Fig. 8 .
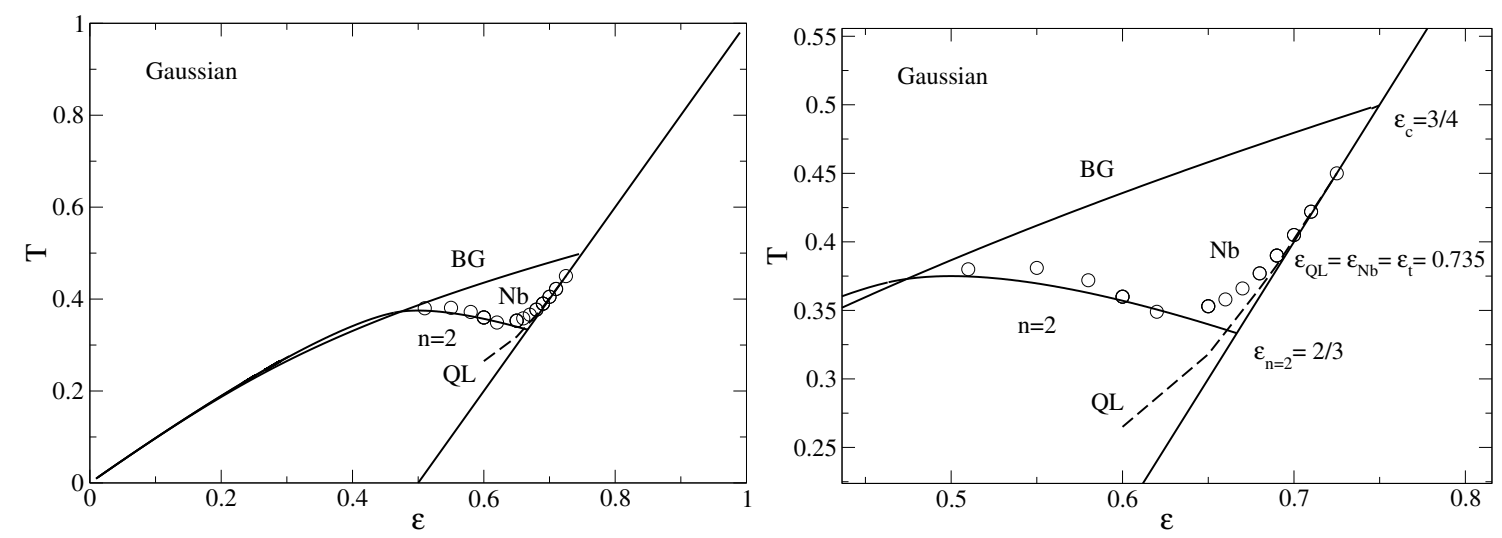

FIG. 8: Caloric curve of the HMF model for a spatially homogeneous Gaussian initial condition (BG: Boltzmann-Gibbs states; bullets: results of $N$-body simulations; dashed line: prediction of the QL theory; $n=2$ : polytropic fit).

In these curves, the solid line denoted BG corresponds to the Boltzmann-Gibbs caloric curve. It describes the statistical equilibrium state of the system, reached for $t \rightarrow+\infty$, as a result of a collisional evolution (finite $N$-effects). Although this curve is not physically 

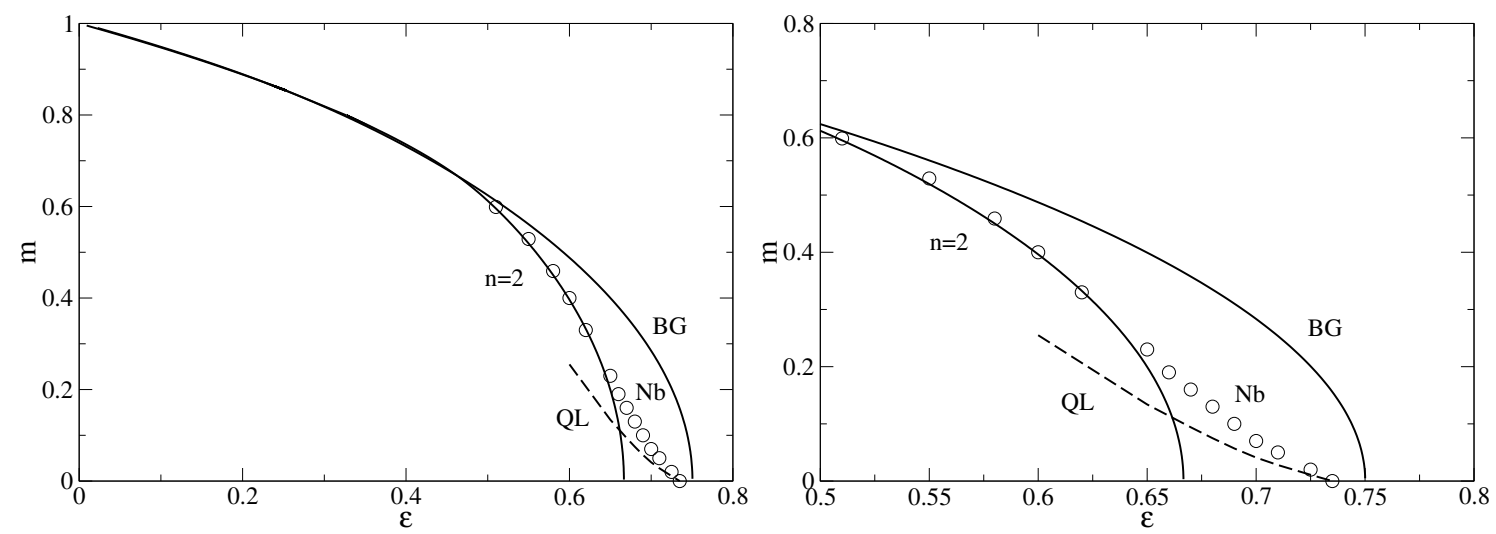

FIG. 9: Magnetization curve of the HMF model for a spatially homogeneous Gaussian initial condition.

relevant to interpret QSSs, that are out-of-equilibrium structures, it is plotted for comparison. It also determines the domain of stability/instability of the initial condition that is a homogeneous Gaussian distribution.

The bullets are the results of direct $N$-body simulations. They form the "experimental" caloric curve. It is made of QSSs reached as a result of a violent collisionless relaxation (Vlasov dynamics). This is the curve that we have to explain. This curve presents several striking features that were considered as a "surprise" in the early works on the HMF model [5, 29]: (i) it differs from the BG caloric curve for intermediate energies; (ii) it presents a region of negative specific heats (unlike the BG caloric curve); (iii) the system remains homogeneous (non-magnetized) slightly below the BG critical energy $\epsilon_{c}=3 / 4$. Although many things have been understood on the physics of systems with long-range interactions [14] since the original works on the HMF model (in particular the difference between QSSs and true equilibrium states), we believe that the HMF model has still not revealed all his secrets. In particular, the precise nature of the QSSs, the reason for the region of negative specific heats, and the shift of the transition point are still not clearly understood. These are the questions that initiated the topic more than 20 years ago and that still haven't found a definitive answer. We stress that the caloric curve of Fig. 8 cannot be explained by Lynden-Bell's theory, as pointed out in our previous work [11]. ${ }^{14}$ We shall interpret this

14 The success or failure of the Lynden-Bell theory depends on the initial condition 30. There are cases where the Lynden-Bell prediction works very well (see Refs. 9, 10] and Fig. 36 of [11]) and cases, such 
curve in relation to the QL theory and to polytropic (Tsallis) distributions.

The dashed line corresponds to the prediction of the QL theory. It is obtained by solving the diffusion equation (71) until its convergence towards a steady state (expected to represent the angle-averaged QSS). From the obtained velocity distribution $f_{0}(p)$, we can compute the kinetic temperature $T$ from Eq. (56) and, using Eq. (70), we can obtain the corresponding magnetization $m$.

The solid line denoted " $n=2$ " corresponds to the caloric curve obtained by assuming that the QSSs are polytropes of index $n=2$. This caloric curve exhibits a region of negative specific heats with $C_{\text {kin }}=-5 / 2$. We emphasize that we do not have any theory to predict why the QSSs should be polytropic (Tsallis) distributions and why their index should be $n=2$ for a Gaussian initial distribution. However, as shown in [11], and confirmed below, polytropes of index $n=2$ give a remarkable fit to the QSSs for a wide range of energies.

Let us now describe the curves of Figs. 8 and 9. For $\epsilon>\epsilon_{\mathrm{c}}=3 / 4=0.75$, the homogeneous Gaussian distribution is stable (dynamically and thermodynamically), so the system does not evolve (even on a collisional timescale). For $\epsilon<\epsilon_{\mathrm{c}}$, the homogeneous Gaussian distribution is dynamically (Vlasov) unstable and the system rapidly evolves towards a QSS. The $N$ body simulations show that the QSS is homogeneous for $\epsilon_{\mathrm{Nb}}<\epsilon<\epsilon_{\mathrm{c}}$ and that it becomes magnetized for $\epsilon<\epsilon_{\mathrm{Nb}}$ with $\epsilon_{\mathrm{Nb}} \simeq 0.735$ (this is most clearly seen on the magnetization curve of Fig. 91). The QL theory predicts that the QSS is homogeneous for $\epsilon_{\mathrm{QL}}<\epsilon<\epsilon_{\mathrm{c}}$ and that it becomes magnetized for $\epsilon<\epsilon_{\mathrm{QL}}$ with $\epsilon_{\mathrm{QL}} \simeq 0.735$. We observe that $\epsilon_{\mathrm{QL}} \simeq \epsilon_{\mathrm{Nb}}$ in $\operatorname{good}$ approximation so the QL theory correctly predicts the energy threshold $\epsilon_{t} \simeq \epsilon_{\mathrm{QL}} \simeq \epsilon_{\mathrm{Nb}} \simeq$ 0.735 below which the QSSs become magnetized (i.e. depart from the line of homogeneous states). This is a non-trivial prediction of the QL theory. The polytropic caloric curve of index $n=2$ exhibits a transition energy $\epsilon_{n=2}=2 / 3 \simeq 0.666$ but this is not a prediction since we have no way to say a priori why the polytropic index should be $n=2$ without doing a $N$-body numerical simulation and making a fit by a polytrope [11]. By contrast, the result of the QL theory is a prediction since it does not rely on a direct $N$-body simulation of the

as the one we are investigating here, where it does not work 11. We can state that the Lynden-Bell prediction does not work without making explicit calculations (which would require a difficult numerical work) because, as found in [11] and discussed further in Sec. VB the QSSs have a compact support (they can be fitted by polytropes of index $n=2$ ) while the Lynden-Bell distribution has no cut-off in energy. 
HMF model; it is directly obtained from the diffusion equation (71). ${ }^{15}$ On the other hand, the transition energy $\epsilon_{\mathrm{QL}} \simeq 0.735$ predicted by the $\mathrm{QL}$ theory is in much better agreement with the "experimental" results than the transition energy $\epsilon_{n=2}=2 / 3 \simeq 0.666$ corresponding to the $n=2$ polytropic fit. Indeed, the points of the $N$-body simulation (QSSs) depart from the homogeneous branch $(m=0)$ long before the energy $\epsilon_{n=2}$ (the $N$-body simulation shows that the transition between un-magnetized and magnetized states occurs at $\epsilon_{\mathrm{Nb}}=0.735$ which is sensibly larger than $\left.\epsilon_{n=2}=2 / 3 \simeq 0.666\right)$. In addition, for $\epsilon>\epsilon_{n=2}$ the caloric curve predicted by the QL theory gives a much better agreement with the results of the $N$-body simulation than the caloric curve of a $n=2$ polytrope that predicts $m=0$. By contrast, for $\epsilon<\epsilon_{n=2}$ the situation is reversed. In that case, the $n=2$ polytropes provide a much better agreement with the $N$-body simulation than the QL theory. This is revealed not only by the values of the magnetization and kinetic temperature that are very close to the numerical ones, but also by the distribution function itself as discussed in Sec. VB. Therefore, we conclude that the QL theory works well close to the critical energy $\epsilon_{\mathrm{c}}$ (in particular, it is able to predict the shift $\Delta \epsilon=\epsilon_{t}-\epsilon_{c}=-0.015$ of the transition energy from un-magnetized to magnetized QSSs) while the polytropic fit works well at lower energies $\epsilon<\epsilon_{n=2}$ (in particular it is able to account for the region of negative specific heats). These results, which were foreseen in [11], represent an improvement in the understanding of the caloric curve of Fig. 8. However, everything is still not understood. First, we do not know why the QSSs are so well-fitted by polytropic (Tsallis) distributions of index $n=2$ (this is a property of incomplete mixing but it is not clear how one can predict the efficiency of mixing [7]). Second, even if the QL theory is qualitatively correct close to $\epsilon_{c}$, it does not give a perfect agreement with the results of the $N$-body simulations.

\section{B. Velocity distribution functions}

In this section, we compare the velocity distribution functions obtained from the $N$-body simulations, the QL theory, and the $n=2$ polytropic fit for two different energies.

We first consider an energy $\epsilon=0.69$ close to the critical point $\epsilon_{\mathrm{c}}=3 / 4=0.75$. The

${ }^{15}$ We note that it is necessary to solve a dynamical equation, which is a smoothing (or a coarse-graining) of the Vlasov equation, in order to predict the QSS. This confirms the claim made in [7] concerning the importance of the dynamics. 


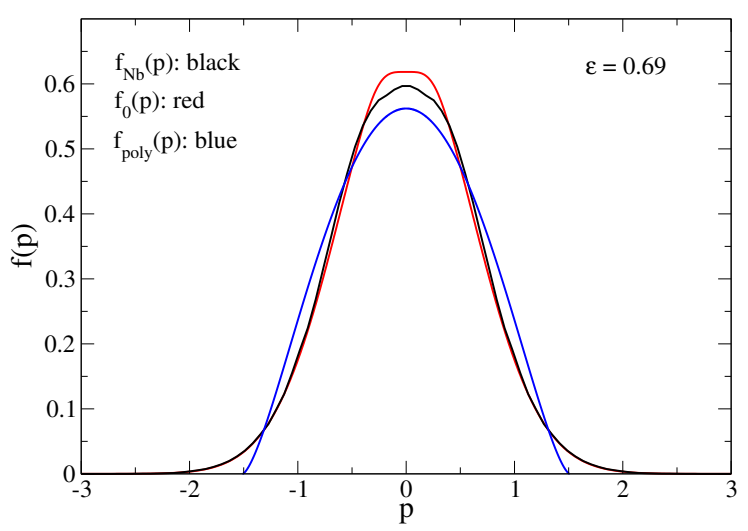

FIG. 10: Velocity distribution of the QSS for $\epsilon=0.69$ (black: $N$-body simulation; red: QL theory; blue: polytrope $n=2$ ).

velocity distributions are plotted in Fig. 10. As already discussed in Sec. IVB, we find a relatively good agreement between the $\mathrm{QL}$ theory and the $N$-body simulation. This is of course consistent with the discussion of Sec. $\mathrm{VA}$ where we showed that the QL theory works well for weakly inhomogeneous systems. We note that the tail of the distribution function has almost not evolved and coincides with the initial Gaussian distribution. Only the core of the distribution has changed in order to make the system dynamically stable. The relatively good agreement between the QL theory and the $N$-body simulation is confirmed by the values of the kinetic temperature and magnetization. We find $T_{\mathrm{QL}}=0.383$ and $m_{\mathrm{QL}}=0.057$ to be compared with $T_{\mathrm{Nb}}=0.390$ and $m_{\mathrm{Nb}}=0.1$. On the other hand, the fit by a polytrope $n=2$ is not good. ${ }^{16}$ The main reason is that the velocity distribution of the polytrope $n=2$ has a compact support that is in contradiction with the infinite extension of the Gaussian tail of the QSS. The disagreement between the $n=2$ polytrope and the $N$-body simulation is confirmed by the values of the kinetic temperature and magnetization. We find $T_{\text {poly }}=0.38$ and $m_{\text {poly }}=0$ (homogeneous) to be compared with $T_{\mathrm{Nb}}=0.390$ and $m_{\mathrm{Nb}}=0.1$ (inhomogeneous).

We now consider a lower energy $\epsilon=0.6$. The velocity distributions are plotted in Fig. 11. In that case, the situation is reversed. We observe a remarkable agreement between the velocity distribution of the QSS reached in the $N$-body simulation and the velocity

${ }^{16}$ For $\epsilon=0.69>\epsilon_{n=2}=2 / 3 \simeq 0.666$ the polytrope $n=2$ is homogeneous so it is not necessary to average the polytropic distribution function over the angles. 


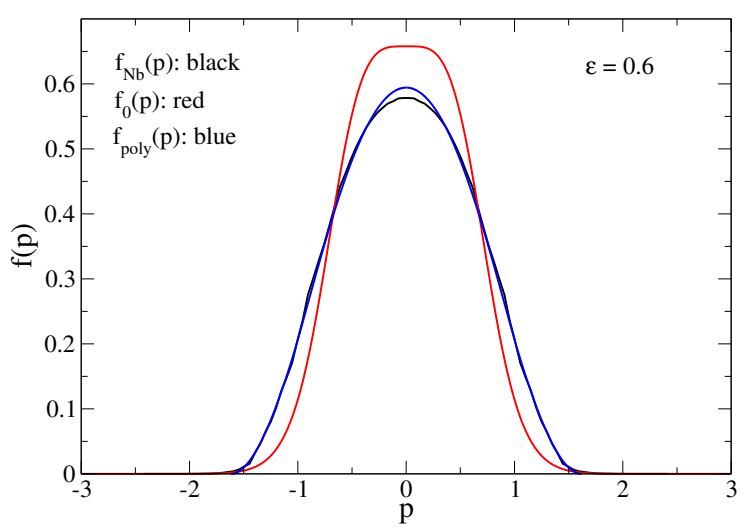

FIG. 11: Velocity distribution of the QSS for $\epsilon=0.6$ (black: $N$-body simulation; red: QL theory; blue: polytrope $n=2$ ).

distribution of a polytrope $n=2 .{ }^{17}$ The distribution function has substantially evolved from the initial Gaussian distribution and has acquired a compact support, which is a property of polytropic (Tsallis) distribution with index $n \geq 1 / 2$ [11, 16]. As discussed in [11], this "confinement" is a manifestation of an incomplete relaxation (the distribution function predicted by the Lynden-Bell theory, which corresponds to the most mixed state, never has a compact support). The excellent agreement between the $N$-body simulation and the $n=2$ polytropic fit is confirmed by the values of the kinetic temperature and magnetization. We find $T_{\text {poly }}=0.357$ and $m_{\text {poly }}=0.396$ to be compared with $T_{\mathrm{Nb}}=0.360$ and $m_{\mathrm{Nb}}=0.40$. On the other hand, the prediction of the QL theory is not good. The main reason is that this distribution has a Gaussian tail, similarly to the initial condition, that is in contradiction with the compact support of the QSS. The disagreement between the QL theory and the $N$-body simulation is confirmed by the values of the kinetic temperature and magnetization. We find $T_{\mathrm{QL}}=0.265$ and $m_{\mathrm{QL}}=0.255$ to be compared with $T_{\mathrm{Nb}}=0.360$ and $m_{\mathrm{Nb}}=0.40$.

At even lower energies (typically $\epsilon<0.55$ ), our study in [11] shows that the system takes a core-halo structure. The core corresponds to the pure polytrope $n=2$ but it is now surrounded by a halo of particles. We note that the presence of the halo does not

\footnotetext{
${ }^{17}$ For $\epsilon=0.6<\epsilon_{n=2}=2 / 3 \simeq 0.666$ the polytrope $n=2$ is inhomogeneous and, in order to obtain the velocity distribution $f(p)$, we have integrated the polytropic distribution function $f(\theta, p)$ over the angles using the theoretical results obtained in [11, 16]. As shown in [11, the excellent agreement between the polytrope $n=2$ and the QSS is also valid for the complete distribution function $f(\theta, p)=f(\epsilon)$.
} 
significantly affect the value of the kinetic temperature and magnetization of the QSS since a pure $n=2$ polytrope still gives a rather good fit to the caloric curve and magnetization curve at low energies (see Figs. 8 and 9).

\section{Summary and discussion}

In summary, for a homogeneous Gaussian initial distribution, we have the following results. For $\epsilon>\epsilon_{c}$, the system remains in the homogeneous Gaussian distribution which is stable. For $\epsilon_{n=2}<\epsilon<\epsilon_{c}$, the QL theory works reasonably well. In particular, it is able to predict the energy $\epsilon_{t} \simeq 0.735$ marking the transition between unmagnetized and magnetized QSSs. More precisely (i) for $\epsilon_{t}<\epsilon<\epsilon_{c}$, the system achieves a homogeneous modified Gaussian distribution with a flat core; (ii) for $\epsilon_{n=2}<\epsilon<\epsilon_{t}$, the system achieves an inhomogeneous modified Gaussian distribution with a flat core. For lower energies, the QL theory does not work. In that case, we observe that the QSSs are remarkably well fitted by polytropic (Tsallis) distributions with index $n=2$. They have compact support. For $\epsilon<\epsilon_{n=2}$, the stable polytropic distributions are inhomogeneous. These polytropic distributions are able to account for the region of negative specific heats in the out-of-equilibrium caloric curve, unlike the Boltzmann distribution and (presumably) the Lynden-Bell distribution, and unlike the QL theory. At even lower energies, the system takes a core-halo structure with a polytropic core and a halo of particles.

The QL theory and the polytropic fit have different, but well-defined, domains of validity. This may be qualitatively understood as follows. The QL theory is based on the assumption that the system remains slightly inhomogeneous during its evolution from the initial condition to the QSS (weak mixing). Therefore, the QL theory is expected to be valid close to the instability threshold $\epsilon_{c}$. In that case, the distribution function does not substantially change from the initial condition. It just changes a little in a manner to become dynamically (Vlasov) stable. ${ }^{18}$ As we have seen, this change only concerns the core of the distribution

18 This is the stabilization mechanism of the QL theory. The initial condition $f_{0}(p, t=0)$ is dynamically unstable meaning that the complex pulsation $\omega_{I}(t=0)$ is strictly positive. Qualitatively, this is because the velocity dispersion (kinetic temperature) is too low [see Eq. [67]]. The initially positive complex pulsation $\omega_{I}(t=0)>0$ causes the distribution function to diffuse [see Eqs. 771 and 772]. By diffusing, the distribution function spreads and its velocity dispersion increases. As a result, the complex pulsation $\omega_{I}(t)$ decreases [see Eq. 655]. The diffusion stops when $\omega_{I}(t)$, hence $D(p, t)$, vanishes leading the system 
which becomes flatter and wider ("hotter"). The tail of the distribution does not change and remains Gaussian, as the initial state. When we depart significantly from the instability threshold, the evolution of the system is expected to be more violent and the system is expected to be strongly inhomogeneous at least during the early stage of its evolution (strong mixing). In that case, the distribution function is expected to change substantially from the initial condition. This is the regime where the Lynden-Bell [8] theory should apply in principle. However, it turns out that, in many cases, the relaxation to the Lynden-Bell statistical equilibrium state is incomplete [7]. In case of incomplete relaxation, polytropic distributions may provide a good fit of the QSS because they have a compact support that is typical of an incomplete relaxation (in case of incomplete relaxation high energy states are less populated than what is predicted from the statistical theory of Lynden-Bell) [11, 16, 30]. This is probably why the polytropic fit works well for low values of the energy. In that case, the distribution function changes from an initial Gaussian to a polytrope with a compact support.

\section{THE CASE OF A HOMOGENEOUS SEMI-ELLIPTICAL INITIAL CONDI- TION}

In this section, we show how the previous results are modified when we consider a homogeneous semi-elliptical initial condition (homogeneous polytrope of index $n=1$ ) instead of a homogeneous Gaussian initial condition.

\section{A. The $N$-body simulations and the comparison with the results of the diffusion} equation

In Fig. 12, we plot the temporal evolution of the magnetization obtained from the $N$ body simulations and by solving the diffusion equation of the QL theory for $\epsilon=0.61$. The interpretation is similar to that given previously for the initial Gaussian distribution. For $\epsilon=0.61$, the final magnetization predicted by the $\mathrm{QL}$ theory $\left(m_{\mathrm{QL}}=0.05\right)$ is relatively close to the value obtained from the $N$-body simulation $\left(m_{\mathrm{Nb}}=0.07\right)$ by averaging over the oscillations.

to a Vlasov stable, or marginally stable, QSS. 

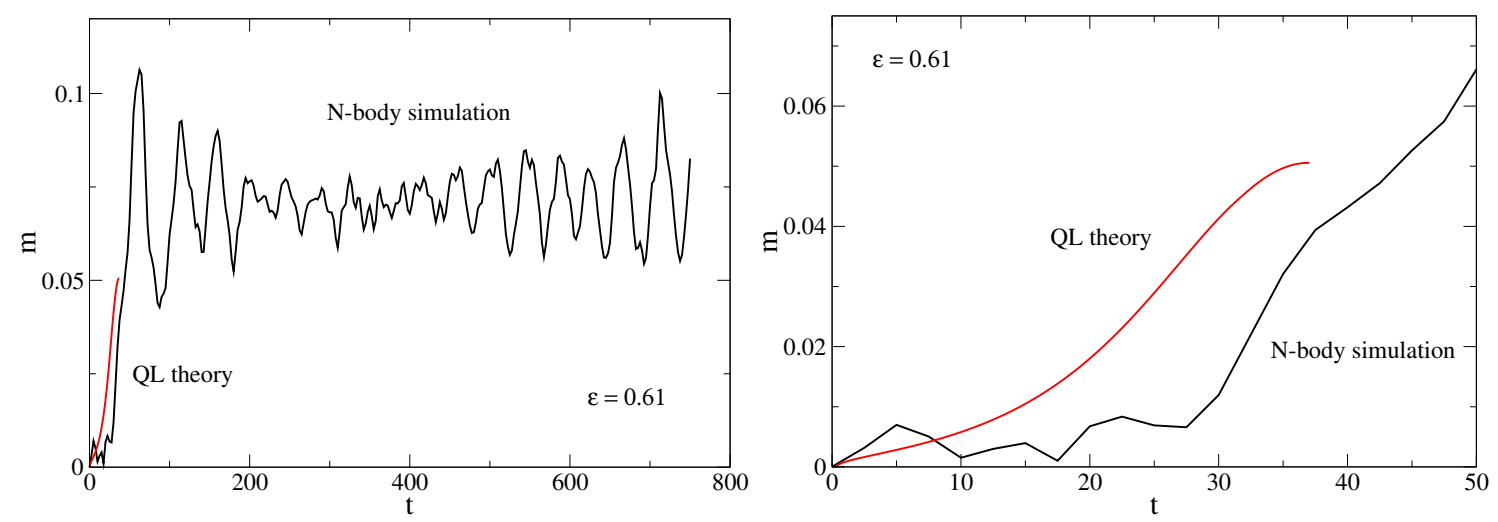

FIG. 12: Magnetization as a function of time at energy $\epsilon=0.61$ for a homogeneous semi-elliptical velocity initial distribution. In order to optimize the agreement between the $N$-body simulation (black curve) and the QL theory (red curve) for the chosen value of $N=2^{18}$, we have solved the diffusion equation with $\chi(0)=10^{-6}$. As in the Gaussian case, a different choice of $\chi(0)$ affects the time scale, but not the final distribution or the final value of the magnetization.

In Fig. 13, we compare the velocity distribution function of the QSS reached in the $N$-body simulation and the final distribution function of the diffusion equation. Like the initial distribution function, the final distribution function has a compact support (actually, the distribution function keeps a compact support at all times). We consider two energies $\epsilon=0.61$ and $\epsilon=0.59$. For $\epsilon=0.61$, which is close to the instability threshold $\epsilon_{c}^{*}=\epsilon_{n=1}=$ $5 / 8=0.625$ of the homogeneous semi-elliptical distribution ( $n=1$ polytrope), the agreement between the QL theory and the $N$-body simulation is good except in the central region (and to a lesser extent close to the maximum velocity). We note that the distribution function has not changed much as compared to the initial semi-elliptical distribution. Only the core of the distribution and the region close to the maximum velocity have changed in order to make the distribution dynamically stable (without this small change, the distribution would be unstable). In particular, the core of the distribution becomes flatter and wider ("hotter"). For the lower energy $\epsilon=0.59$, the disagreement is more pronounced everywhere. The distribution function in the $N$-body simulation has substantially evolved from the initial semi-elliptical distribution and has spread (while keeping a compact support) contrary to the distribution of the QL theory. These results are similar to those obtained in the case of a Gaussian initial distribution. We note, however, that in the semi-elliptical case, close to 

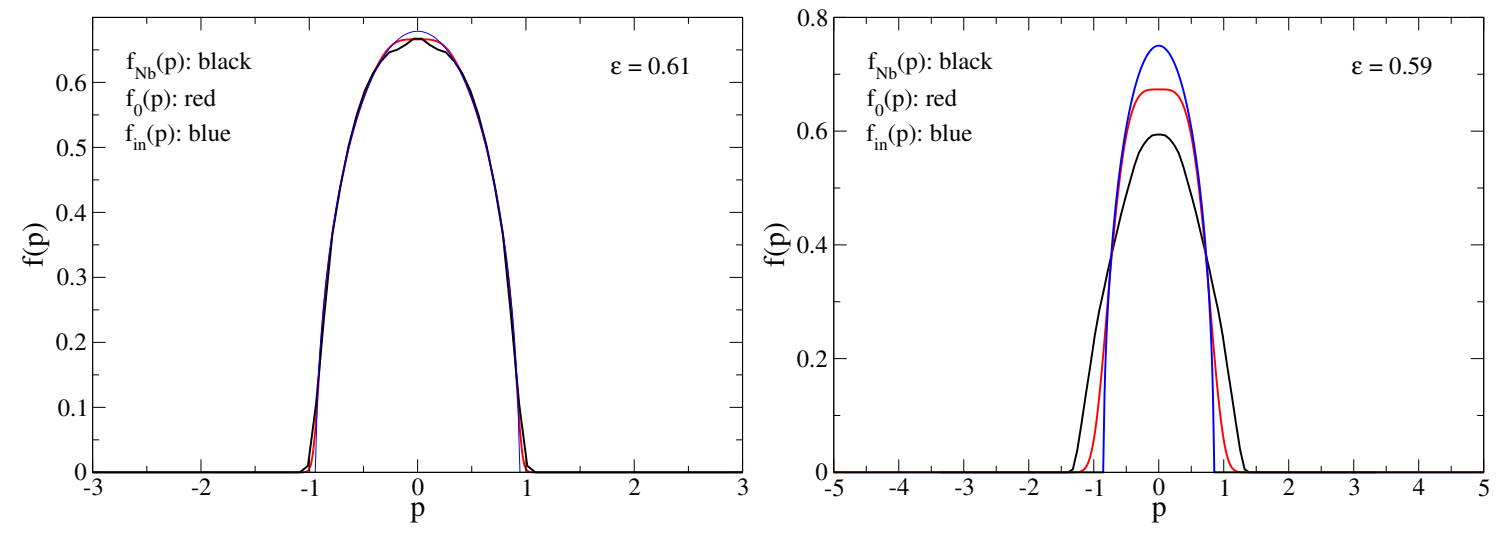

FIG. 13: The final distribution function $f_{0}(p)$ of the diffusion equation and the velocity distribution $f_{\mathrm{Nb}}(p)$ of the QSS of the $N$-body simulation, starting from a homogeneous semi-elliptical initial distribution with energy $\epsilon=0.61$ (left pannel) and $\epsilon=0.59$ (right pannel). We have also plotted the initial condition for comparison. We emphasize that the initial distribution is Vlasov unstable while the final distribution is Vlasov stable even when the profile does not seem to have changed a lot (left panel).

the instability threshold $\epsilon_{c}^{*}$, the distribution function changes from the initial distribution function not only in the core but also in the tail, close to the maximum velocity. By contrast, in the Gaussian case, close to the instability threshold $\epsilon_{c}$, the tail of the distribution almost does not change from the initial condition.

\section{B. Caloric and magnetization curves}

In Fig. 14, we plot the kinetic temperature and the magnetization of the final distribution of the QL theory and of the $N$-body simulation as a function of the energy. The two curves approach each other close to the instability threshold. In particular, the QL theory is able to predict the energy of the out-of-equilibrium phase transition from the unmagnetized state $(m=0)$ to the magnetized state $(m \neq 0)$ as discussed below.

In Figs. 15 and 16, we compare the caloric curve $T(\epsilon)$ and the magnetization curve $m(\epsilon)$ obtained from direct $N$-body simulations of the HMF model, starting from a homogeneous semi-elliptical distribution, with the prediction of the QL theory and with the fit corresponding to a polytrope $n=1$ considered in our previous papers [11, 16]. The bullets are 

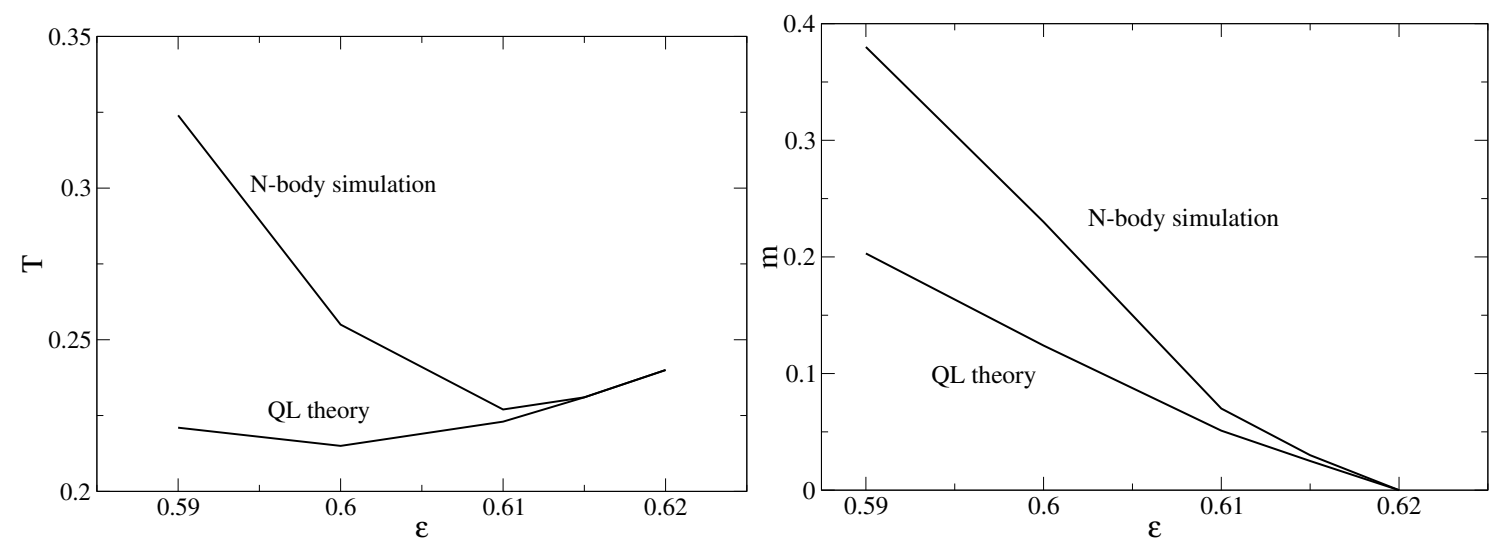

FIG. 14: The kinetic temperature and the magnetization of the stationary state reached by the diffusion equation of the QL theory and that of the QSS reached by the $N$-body simulation, as a function of the energy.

the results of direct $N$-body simulations. The dashed line corresponds to the prediction of the QL theory. The solid line denoted " $n=1$ " corresponds to the caloric curve obtained by assuming that the QSSs are polytropes of index $n=1$. This caloric curve exhibits a region of negative specific heats with $C_{\text {kin }}=-1 / 2$. As shown in [11], and confirmed below, polytropes of index $n=1$ give a remarkable fit to the QSSs for a wide range of energies.
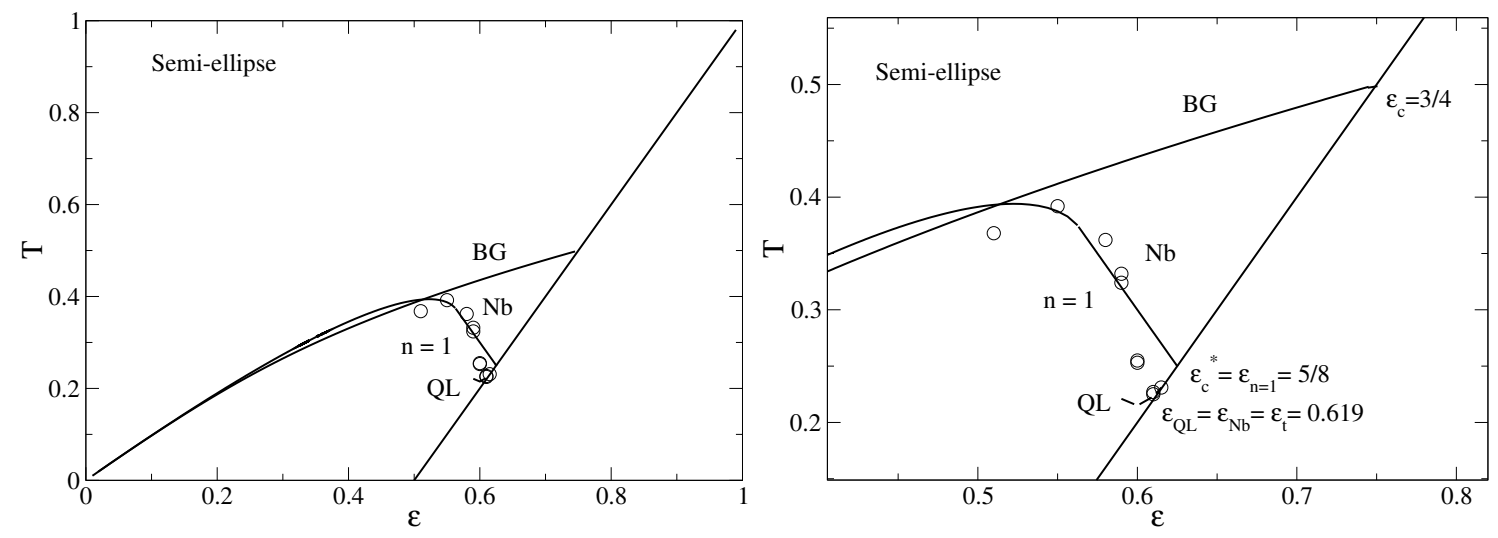

FIG. 15: Caloric curve of the HMF model for a spatially homogeneous semi-elliptical initial condition (BG: Boltzmann-Gibbs states; bullets: results of $N$-body simulations; dashed line: prediction of the QL theory; $n=1$ : polytropic fit).

The initial condition, which is a spatially homogeneous semi-elliptical distribution, corresponds to a polytrope of index $n=1$. Therefore, for $\epsilon>\epsilon_{\mathrm{c}}^{*}=\epsilon_{n=1}=5 / 8=0.625$, 

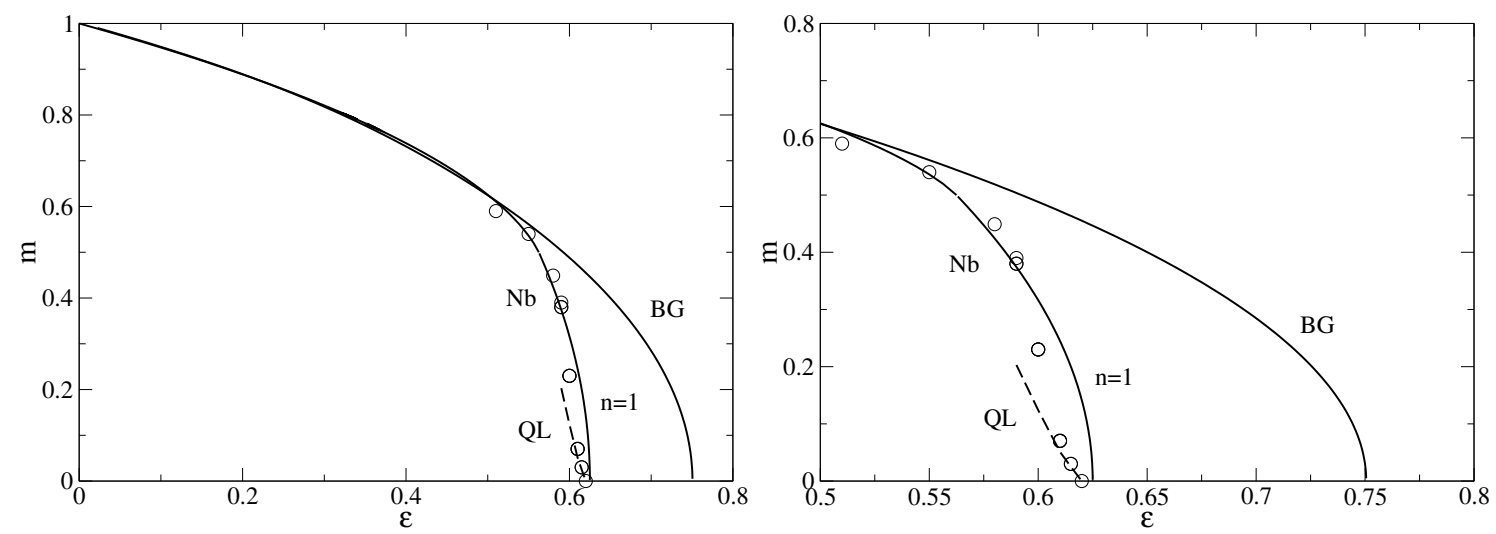

FIG. 16: Magnetization curve of the HMF model for a spatially homogeneous semi-elliptical initial condition.

the homogeneous semi-elliptical distribution is dynamically stable and the system does not evolve on a collisionless timescale. For $\epsilon<\epsilon_{\mathrm{c}}^{*}$, the homogeneous semi-elliptical distribution is dynamically (Vlasov) unstable and the system rapidly evolves towards a QSS.

Slightly below $\epsilon_{\mathrm{c}}^{*}$, the QL theory is in good agreement with $N$-body simulations. It predicts that the QSS remains homogeneous (non magnetized) for $\epsilon_{Q L} \simeq 0.619<\epsilon<\epsilon_{\mathrm{c}}^{*}$ and that it becomes inhomogeneous for $\epsilon<\epsilon_{Q L}$ (the value $\epsilon_{Q L} \simeq 0.619$ has been extrapolated from the magnetization curve of Fig. 14). The transition energy $\epsilon_{Q L} \simeq 0.619$ predicted by the QL theory is in good agreement with the value $\epsilon_{\mathrm{Nb}} \simeq 0.619$ obtained from the $N$ body simulations (also extrapolated from the magnetization curve of Fig. 14). Therefore, for a homogeneous semi-elliptical initial condition, the out-of-equilibrium transition energy is $\epsilon_{t} \simeq \epsilon_{\mathrm{QL}} \simeq \epsilon_{\mathrm{Nb}} \simeq 0.619$ (it can be compared to the value $\epsilon_{t} \simeq 0.735$ obtained for a homogeneous Gaussian initial condition). For $\epsilon_{t}<\epsilon<\epsilon_{\mathrm{c}}^{*}$, the distribution is not a homogeneous polytrope of index $n=1$ since this distribution is unstable. The observed distribution of the QSS is close to the distribution predicted by the QL theory as analyzed in Sec. VIC,

For smaller energies, the QL theory does not give a good agreement with the $N$-body simulations anymore. In that case, we observe (in agreement with our earlier paper [11]) that the QSS is well-fitted by an inhomogenous $n=1$ polytrope. This is revealed not only by the values of the magnetization and kinetic temperature that are very close to the numerical ones, but also by the distribution function itself as discussed in Sec. VIC. We 
have, however, no theory to justify why the $n=1$ polytropic fit works so well.

In conclusion, the QL theory works well close to the critical energy $\epsilon_{\mathrm{c}}^{*}$ (in particular it is able to predict the shift $\Delta \epsilon=\epsilon_{t}-\epsilon_{c}^{*}=-0.006$ of the transition energy from un-magnetized to magnetized QSSs) while the polytropic fit works well at lower energies (in particular it is able to account for the region of negative specific heats). These conclusions are similar to those obtained for a spatially homogeneous Gaussian initial condition. We note, however, that the shift $\Delta \epsilon=\epsilon_{t}-\epsilon_{c}^{*}=-0.006$ obtained in the semi-elliptical case is smaller than the shift $\Delta \epsilon=\epsilon_{t}-\epsilon_{c}=-0.015$ obtained in the Gaussian case. We also note that, in the semi-elliptical case, the transition energy $\epsilon_{t} \simeq 0.619$ predicted by the $\mathrm{QL}$ theory is below the transition energy $\epsilon_{\mathrm{c}}^{*}=\epsilon_{n=1}=5 / 8=0.625$ corresponding to $n=1$ polytropes while in the Gaussian case, the transition energy $\epsilon_{t} \simeq 0.735$ predicted by the QL theory is above the transition energy $\epsilon_{n=2}=2 / 3 \simeq 0.666$ corresponding to $n=2$ polytropes. Therefore, the fact that we reach similar conclusions in these two different situations is encouraging.

\section{Velocity distribution functions}

In this section, we compare the velocity distribution functions obtained from the $N$-body simulations, the QL theory, and the $n=1$ polytropic fit for two different energies.

We first consider an energy $\epsilon=0.61$ close to the critical point $\epsilon_{\mathrm{c}}^{*}=5 / 8=0.625$. The velocity distributions are plotted in Fig. 17. As already discussed in Sec. VIA, we find a relatively good agreement between the QL theory and the $N$-body simulation. This relatively good agreement is confirmed by the values of the kinetic temperature and magnetization. We find $T_{\mathrm{QL}}=0.223$ and $m_{\mathrm{QL}}=0.05$ to be compared with $T_{\mathrm{Nb}}=0.227$ and $m_{\mathrm{Nb}}=0.07$. On the other hand, the fit by an inhomogeneous polytropic distribution of index $n=1$ is not good. Although it has a compact support, it is more spread than the QSS obtained from the $N$-body simulation. The disagreement between the $n=1$ polytrope and the $N$-body simulation is confirmed by the values of the kinetic temperature and magnetization. We find $T_{\text {poly }}=0.28$ and $m_{\text {poly }}=0.245$ to be compared with $T_{\mathrm{Nb}}=0.227$ and $m_{\mathrm{Nb}}=0.07$.

We now consider a lower energy $\epsilon=0.59$. The velocity distributions are plotted in Fig. 18. In that case, the situation is reversed. We observe a remarkable agreement between the velocity distribution of the QSS obtained from the $N$-body simulation and the velocity 


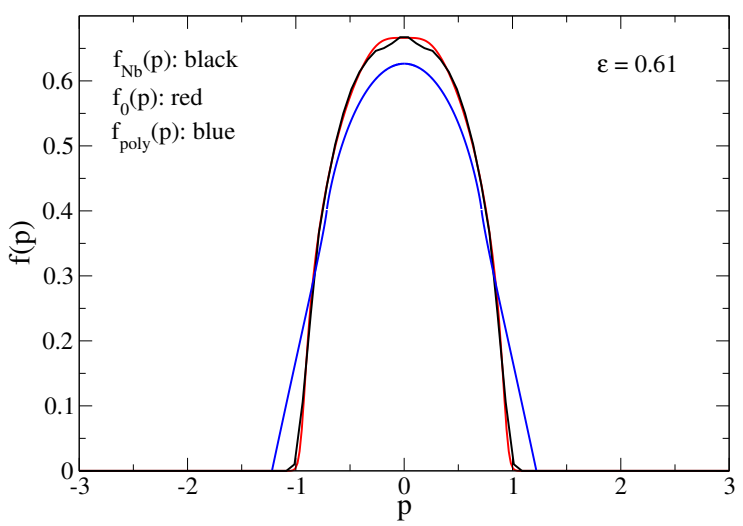

FIG. 17: Velocity distribution of the QSS for $\epsilon=0.61$ (black: $N$-body simulation; red: QL theory; blue: polytrope $n=1$ ).

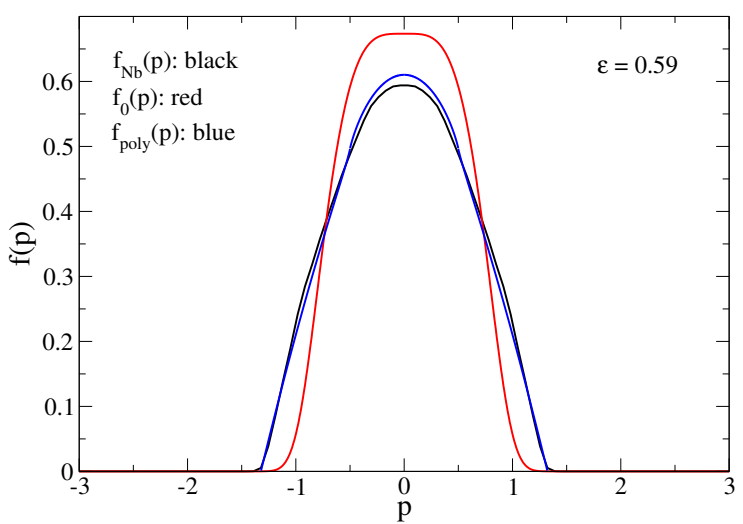

FIG. 18: Velocity distribution of the QSS for $\epsilon=0.59$ (black: $N$-body simulation; red: QL theory; blue: polytrope $n=1$ ).

distribution of a polytrope $n=1 .^{19}$ This is confirmed by the values of the kinetic temperature and magnetization. We find $T_{\text {poly }}=0.32$ and $m_{\text {poly }}=0.374$ to be compared with $T_{\mathrm{Nb}}=0.324$ and $m_{\mathrm{Nb}}=0.38$. On the other hand, the prediction of the QL theory is not good. This distribution has not spread enough from the initial condition (see Fig. 13). The disagreement between the QL theory and the $N$-body simulation is confirmed by the values of the kinetic temperature and magnetization. We find $T_{\mathrm{QL}}=0.22$ and $m_{\mathrm{QL}}=0.203$ to be compared with $T_{\mathrm{Nb}}=0.324$ and $m_{\mathrm{Nb}}=0.38$.

${ }^{19}$ As shown in [11, the excellent agreement between the polytrope $n=1$ and the QSS is also valid for the complete distribution function $f(\theta, p)=f(\epsilon)$. 
At even lower energies (e.g. $\epsilon=0.55$ ), our study in [11] shows that the system takes a core-halo structure. The core corresponds to the pure polytrope $n=1$ but it is now surrounded by a halo of particles.

\section{Summary and discussion}

In summary, for a homogeneous semi-elliptical initial distribution, we have the following results. For $\epsilon>\epsilon_{c}^{*}=\epsilon_{n=1}=0.625$, the system remains in the homogeneous semi-elliptical distribution which is stable. Close to $\epsilon_{c}^{*}$, the QL theory works reasonably well. In particular, it is able to predict the energy $\epsilon_{t} \simeq 0.619$ marking the transition between unmagnetized and magnetized QSSs. For lower energies, the QL theory does not work. In that case, we observe that the QSSs are remarkably well fitted by inhomogeneous polytropic distributions (Tsallis distributions) with index $n=1$. These polytropic distributions are able to account for the region of negative specific heats in the out-of-equilibrium caloric curve, unlike the Boltzmann and Lynden-Bell distributions. At even lower energies, the system takes a core-halo structure with a polytropic core and a halo of particles.

Since the initial condition is a spatially homogeneous polytrope of index $n=1$, and since it appears that the QSSs are often well-fitted by inhomogeneous polytropes of index $n=1$, we could expect that the QSS could be fitted by an inhomogeneous polytrope of index $n=1$ as soon as $\epsilon<\epsilon_{c}^{*}=\epsilon_{n=1}$. Actually, this is not the case. There is a small range of energies $\left[\epsilon_{t}, \epsilon_{c}^{*}\right]$ where the QSS remains homogeneous. Within this small range of energies, the QL theory works relatively well, unlike the polytropic fit. Therefore, even if the caloric curves differ in the detail, the results obtained for a semi-elliptical initial distribution are relatively similar to those obtained for a Gaussian initial distribution. In consequence, the phenomenology that we have described seems to be relatively general despite the fact that the nature of the QSS strongly depends on the initial condition.

A striking and mysterious outcome of our study (see also [11, 16]) is the finding that, in many cases, the QSSs are well fitted by polytropic (Tsallis) distributions. The value of the polytropic index depends on the class of initial condition. For the semi-elliptical initial distribution, which is a polytrope of index $n=1$, the polytrope that fits the QSS has the same index as the initial state, i.e., $n=1$. Although not considered in the present paper, we found in [11] that for the waterbag initial distribution, which is a polytrope of index 
$n=1 / 2$, the polytrope that fits the QSS also has the same index, i.e., $n=1 / 2$. By contrast, for the Gaussian initial distribution, which can be viewed as a polytrope of index $n=\infty$, the polytrope that fits the QSS has a different index, $n=2$. We have no theory to explain these striking results. However, they confirm that polytropic (Tsallis) distributions can be useful in systems with long-range interaction as claimed in [30].

\section{CONCLUSION}

In this work, we have considered the QSSs of long-range systems, using the paradigmatic HMF model, that are reached when the system is initially in a spatially homogeneous state which is Vlasov unstable. The Vlasov equation admits an infinity of stable stationary states, each of them representing a possible QSS of the $N$-body system. The prediction of the QSS reached by any given initial unstable state is still practically an unsolved problem although progress has been done lately [1-4]. The Lynden-Bell theory [8] is in a sense conceptually satisfying since it is based, as the equilibrium Boltzmann-Gibbs theory, on the assumption of complete mixing of the dynamics. The differences between the Boltzmann theory and the Lynden-Bell theory come from the difference in the number of integrals of motion between the full $N$-body dynamics (collisional) and the Vlasov dynamics (collisionless). In particular, the Vlasov equation conserves an infinite number of Casimir integrals that must be accounted for in the Lynden-Bell theory. This leads to a great difficulty in the concrete application of the Lynden-Bell theory to generic initial conditions but, at least in principle, one has the tool to determine the QSS as a function of the initial state. Unfortunately, the basic assumption of the Lynden-Bell theory, i.e. complete mixing, seems very far from being satisfied in general [7]. Without this starting point, the construction of alternative theories are conceptually more difficult since it is not yet possible to quantify in a sensible way the departure from complete mixing, and even if it were possible, it would still be uncertain how to translate it in a theory of the dynamical behavior of the system. Thus, for the moment one can only resort to semi-empirical approaches. In Ref. [11] we have proposed, in the absence of complete mixing, to substitute the Lynden-Bell entropy with other Casimirs of the Vlasov equation, e.g. the Tsallis entropy leading to polytropic QSSs. This is, however, essentially empirical because we have no way to predict a priori if the QSS will be a polytrope and, if so, what the value of the polytropic index will be. However, the polytropic fit works 
remarkably well for a wide range of energies, sufficiently far from the instability threshold, and the polytropic index appears to be relatively universal [11] for a given class of initial conditions. $^{20}$ Another proposal has been given in Ref. [31] where a core-halo structure of the QSS was hypothesized from the start. We showed in [11] that our approach based on polytropic distributions is consistent with this core-halo approach and generalizes it. At low energies, we find that the QSS has a core-halo structure with a polytropic core surrounded by a halo of particles. In the case of waterbag initial conditions (that correspond to polytropes of index $n=1 / 2$ ) we recover the "uniform" core-halo structure found in [31. ${ }^{21}$ For other initial conditions, we obtain more general "polytropic" core-halo structures. In the present work, we have developed a QL theory that has proven to work with good approximation close to the instability threshold, i.e., in the energy range where the polytropic fit proposed in Ref. [11] is not good. Contrary to time-independent approaches that try to predict the form of the distribution of the QSS either by the optimization of a functional or by an assumption about the form of its analytical expression, the QL theory requires to solve a dynamical (diffusion) equation that smoothes out the Vlasov equation. ${ }^{22}$ The first approaches are implicitly based on the assumption that the evolution of the system is violent and strongly inhomogeneous so that a change of structure of the initial distribution function occurs. The QL theory, on the other hand, is based on the assumption that throughout the transient from the initial distribution to the QSS the distribution remains always almost homogeneous. The first approaches are expected to work if the instability of the initial distribution is strong, i.e., if the initial condition is far from the instability threshold. The QL theory is expected to work if the instability of the initial distribution is weak, i.e., if the initial condition is close to the instability threshold. These general features have been verified in this work.

Summarizing, the dynamical process leading to a QSS seems to be best described by

20 The quality of the polytropic fit is impressive and the universality of the polytropic index for some classes of equivalence of the initial condition ( $n=2$ for Gaussian, $n=1$ for semi-elliptical and $n=1 / 2$ for waterbag) remains relatively mysterious. Also mysterious is the fact that the collisional evolution of the system can be fitted by a sequence of polytropic distributions with a time-dependent index $n(t)$ both in the homogeneous [27] and in the inhomogeneous [11] phase. These exciting results represent some of the last unsolved mysteries of long-range systems.

${ }^{21}$ To some extent, this core-halo structure can be understood from energetic considerations (see Fig. 34 in [11).

${ }^{22}$ Even in the context of the Lynden-Bell theory, it may be necessary to solve a dynamical (relaxation) equation in order to take into account the problem of incomplete relaxation as discussed in [7. 
different approaches depending on the intial state of the system. The Lynden-Bell theory is based on an assumption of efficient mixing (ergodicity) whose validity is hard to establish a priori. The polytropic and core-halo fits seem to work well when the Lynden-Bell theory fails. The quasilinear theory is a fully predictive theory that is valid in a perturbative regime close to the instability threshold. These approaches have complementary domains of validity. Hopefully, in a near future, these methods will be encompassed into a single more general theory.

\section{Appendix A: The decrease of $\omega_{\mathrm{I}}(t)$ with time}

We have seen in the numerical integration of the diffusion equation that the function $f_{0}(p, t)$ keeps approximately its functional form. This can be exploited to give an argument showing that $\omega_{\mathrm{I}}(t)$ decreases monotonically with time. We start from the dispersion relation (49), that for convenience we recall here:

$$
1+\pi \int \mathrm{d} p \frac{p f_{0}^{\prime}(p, t)}{p^{2}+\omega_{\mathrm{I}}^{2}(t)}=0 .
$$

Let us write the function $f_{0}$ at time $t+\mathrm{d} t$ as

$$
f_{0}(p, t+\mathrm{d} t)=\alpha f_{0}(\alpha p, t)+\delta f(p, t)
$$

where $\alpha=1-\xi$ with $\xi \ll 1$, and where we assume that

$$
|\delta f(p, t)| \ll\left|f_{0}(p, t)-\alpha f_{0}(\alpha p, t)\right|
$$

By neglecting the term $\delta f$ in Eq. A2 we obtain $\omega_{\mathrm{I}}(t+\mathrm{d} t)<\omega_{\mathrm{I}}(t)$ as we show below. The fact that $\alpha<1$ is obtained from Eq. (59). Now, writing the dispersion relation (A1) for $f_{0}(p, t+\mathrm{d} t)$ by using Eq. A2 and neglecting $\delta f$, we have

$$
\begin{aligned}
& 1+\pi \int \mathrm{d} p \frac{p f_{0}^{\prime}(p, t+\mathrm{d} t)}{p^{2}+\omega_{\mathrm{I}}^{2}(t+\mathrm{d} t)}=1+\pi \int \mathrm{d} p \frac{\alpha^{2} p f_{0}^{\prime}(\alpha p, t)}{p^{2}+\omega_{\mathrm{I}}^{2}(t+\mathrm{d} t)} \\
= & 1+\pi \int \mathrm{d} p \frac{p f_{0}^{\prime}(p, t)}{\left(p^{2} / \alpha^{2}\right)+\omega_{\mathrm{I}}^{2}(t+\mathrm{d} t)}=0,
\end{aligned}
$$

where the last passage has been obtained by a trivial change of variable in the integral. If $\omega_{\mathrm{I}}(t+\mathrm{d} t)$ is not smaller than $\omega_{\mathrm{I}}(t)$, then $\left(p^{2} / \alpha^{2}\right)+\omega_{\mathrm{I}}^{2}(t+\mathrm{d} t)>p^{2}+\omega_{\mathrm{I}}^{2}(t)$ for any $p$. Considering that the integrand in the dispersion relation is negative definite, we infer that the right-hand side of Eq. (A4) would be necessarily positive. The only way to make it vanish is to have $\omega_{\mathrm{I}}(t+\mathrm{d} t)<\omega_{\mathrm{I}}(t)$. 


\section{Appendix B: Proof of Eq. (63)}

We write $p /\left(p^{2}+\omega_{\mathrm{I}}^{2}(t)\right)=1 / p-\omega_{\mathrm{I}}^{2}(t) /\left(p^{2}+\omega_{\mathrm{I}}^{2}(t)\right)$. Substituting this decomposition in Eq. (49), the first term gives rise to the integral in Eq. (63). For the second term we have

$$
\begin{gathered}
-\pi \omega_{\mathrm{I}}^{2}(t) \int \mathrm{d} p \frac{f_{0}^{\prime}(p, t)}{p\left(p^{2}+\omega_{\mathrm{I}}^{2}(t)\right)}=-\pi \operatorname{sign}\left[\omega_{\mathrm{I}}(t)\right] \int \mathrm{d} x \frac{f_{0}^{\prime}\left(\omega_{\mathrm{I}}(t) x, t\right)}{x\left(x^{2}+1\right)} \\
=-\pi\left|\omega_{\mathrm{I}}(t)\right| \int \mathrm{d} x \frac{f_{0}^{\prime \prime}(0, t)}{x^{2}+1}+O\left(\omega_{\mathrm{I}}^{2}(t)\right)=-\pi^{2}\left|\omega_{\mathrm{I}}(t)\right| f_{0}^{\prime \prime}(0, t)+O\left(\omega_{\mathrm{I}}^{2}(t)\right) .
\end{gathered}
$$

\section{Appendix C: The modified Gaussian}

Here, we show how to solve the system of equations $(74)-76$ and to obtain the values of $A, \beta$ and $p_{1}$ as a function of $\epsilon_{\text {kin. }}$. Using the complementary error function $\operatorname{erfc}(x)$ defined by

$$
\operatorname{erfc}(x)=1-\operatorname{erf}(x)=\frac{2}{\sqrt{\pi}} \int_{x}^{\infty} \mathrm{d} y \mathrm{e}^{-y^{2}},
$$

the three equations become

$$
\begin{aligned}
2 A p_{1} \mathrm{e}^{-\beta \frac{p_{1}^{2}}{2}}+\sqrt{\frac{2 \pi}{\beta}} A \operatorname{erfc}\left(\sqrt{\frac{\beta}{2}} p_{1}\right) & =\frac{1}{2 \pi}, \\
1-\pi \beta \sqrt{\frac{2 \pi}{\beta}} A \operatorname{erfc}\left(\sqrt{\frac{\beta}{2}} p_{1}\right) & =0 \\
A p_{1} \mathrm{e}^{-\beta \frac{p_{1}^{2}}{2}}\left(\frac{p_{1}^{2}}{3}+\frac{1}{\beta}\right)+\sqrt{\frac{\pi}{2 \beta^{3}}} A \operatorname{erfc}\left(\sqrt{\frac{\beta}{2}} p_{1}\right) & =\frac{\epsilon_{\mathrm{kin}}}{2 \pi} .
\end{aligned}
$$

We know that for the energy $\epsilon_{\text {kin }}=1 / 4$, corresponding to the critical point of the model, for which the Gaussian velocity distribution function is marginally stable, the solution of these three equations is $\beta=2, p_{1}=0$ and $A=1 / 2 \pi^{3 / 2}$. Starting from this solution, it is not difficult to find numerically the solution for other values of $\epsilon_{\text {kin }}$. In fact, the system of three equations is of the form

$$
\begin{aligned}
F_{1}\left(A, \beta, p_{1}\right) & =0, \\
F_{2}\left(A, \beta, p_{1}\right) & =0, \\
F_{3}\left(A, \beta, p_{1} ; \epsilon_{\text {kin }}\right) & =0 .
\end{aligned}
$$

It is then possible to obtain closed expressions for $\partial A / \partial \epsilon_{\text {kin }}, \partial \beta / \partial \epsilon_{\text {kin }}$ and $\partial p_{1} / \partial \epsilon_{\text {kin }}$. Then, starting from the known solution for $\epsilon_{\text {kin }}=1 / 4$, one can compute the solutions for other 
values by numerical integration.

[1] Dynamics and thermodynamics of systems with long range interactions, edited by T. Dauxois et al., Lecture Notes in Physics 602, (Springer, 2002).

[2] Dynamics and thermodynamics of systems with long range interactions: Theory and experiments, edited by A. Campa et al., AIP Conf. Proc. 970 (AIP, 2008).

[3] A. Campa, T. Dauxois, D. Fanelli, and S. Ruffo, Physics of Long-Range Interacting Systems (Oxford University Press, 2014).

[4] A. Campa, T. Dauxois, and S. Ruffo, Phys Rep. 480, 57 (2009).

[5] M. Antoni and S. Ruffo, Phys. Rev. E 52, 2361 (1995).

[6] Y. Yamaguchi, J. Barré, F. Bouchet, T. Dauxois, S. Ruffo, Physica A 337, 36 (2004).

[7] P.H. Chavanis, Physica A 365, 102 (2006).

[8] D. Lynden-Bell, MNRAS 136, 101 (1967).

[9] A. Antoniazzi, D. Fanelli, J. Barré, P.H. Chavanis, T. Dauxois, and S. Ruffo, Phys. Rev. E 75, 011112 (2007).

[10] F. Staniscia, P.H. Chavanis, G. de Ninno, and D. Fanelli, Phys. Rev. E 80, 021138 (2009).

[11] A. Campa and P.H. Chavanis, Eur. Phys. J. B 86, 170 (2013).

[12] C. Tsallis, Introduction to Nonextensive Statistical Mechanics (Springer, 2009).

[13] J. Binney and S. Tremaine, Galactic Dynamics (Princeton Series in Astrophysics, 2008).

[14] S. Tremaine, M. Hénon and D. Lynden-Bell, Mon. Not. R. astr. Soc. 219, 285 (1986).

[15] A. Campa and P.H. Chavanis, J. Stat. Mech. 6, 06001 (2010).

[16] P.H. Chavanis and A. Campa, Eur. Phys. J. B 76, 581 (2010).

[17] P.H. Chavanis, AIP Conf. Proc. 970, 39 (2008).

[18] D.R. Nicholson, Introduction to Plasma Theory (Krieger Publishing Company, Florida, 1992).

[19] P.H. Chavanis, Eur. Phys. J. Plus 127, 19 (2012).

[20] P.H. Chavanis, J. Sommeria and R. Robert, Astrophys. J. 471, 385 (1996).

[21] P.H. Chavanis, Mon. Not. R. astr. Soc. 300, 981 (1998).

[22] K. M. Case, Ann. Phys. 7, 349 (1959).

[23] N.G. Van Kampen, Physica 21, 949 (1955).

[24] L. Landau, J. Phys. U.S.S.R. 10, 25 (1946). 
[25] P.H. Chavanis and L. Delfini, Eur. Phys. J. B 69, 389 (2009).

[26] P.H. Chavanis, Eur. Phys. J. B 85, 229 (2012).

[27] A. Campa, P.H. Chavanis, A. Giansanti, and G. Morelli, Phys. Rev. E 78, 040102 (2008).

[28] H. Morita and K. Kaneko, Phys. Rev. Lett. 96, 050602 (2006).

[29] V. Latora, A. Rapisarda, and C. Tsallis, Phys. Rev. E 64, 056134 (2001).

[30] P.H. Chavanis, Eur. Phys. J. B 53, 487 (2006).

[31] R. Pakter and Y. Levin, Phys. Rev. Lett. 106, 200603 (2011). 